\title{
Naïve imitation and partial cooperation in a local public goods model
}

Citation for published version (APA):

Herings, P. J-J., Peeters, R., Tenev, A., \& Thuijsman, F. (2019). Naïve imitation and partial cooperation in a local public goods model. Maastricht University, Graduate School of Business and Economics. GSBE Research Memoranda No. 013 https://doi.org/10.26481/umagsb.2019013

Document status and date:

Published: 25/04/2019

DOI:

10.26481/umagsb.2019013

Document Version:

Publisher's PDF, also known as Version of record

\section{Please check the document version of this publication:}

- A submitted manuscript is the version of the article upon submission and before peer-review. There can be important differences between the submitted version and the official published version of record.

People interested in the research are advised to contact the author for the final version of the publication, or visit the DOI to the publisher's website.

- The final author version and the galley proof are versions of the publication after peer review.

- The final published version features the final layout of the paper including the volume, issue and page numbers.

Link to publication

\footnotetext{
General rights rights.

- You may freely distribute the URL identifying the publication in the public portal. please follow below link for the End User Agreement:

www.umlib.nl/taverne-license

Take down policy

If you believe that this document breaches copyright please contact us at:

repository@maastrichtuniversity.nl

providing details and we will investigate your claim.
}

Copyright and moral rights for the publications made accessible in the public portal are retained by the authors and/or other copyright owners and it is a condition of accessing publications that users recognise and abide by the legal requirements associated with these

- Users may download and print one copy of any publication from the public portal for the purpose of private study or research.

- You may not further distribute the material or use it for any profit-making activity or commercial gain

If the publication is distributed under the terms of Article $25 \mathrm{fa}$ of the Dutch Copyright Act, indicated by the "Taverne" license above, 
P. Jean-Jacques Herings,

Ronald Peeters,

Anastas P. Tenev,

Frank Thuijsman

Naïve imitation and partial cooperation in a local public goods model

RM/19/013

\section{GSBE}

Maastricht University School of Business and Economics

Graduate School of Business and Economics

P.O Box 616

NL- 6200 MD Maastricht

The Netherlands 


\title{
Naïve imitation and partial cooperation in a local public goods model
}

\author{
P. Jean-Jacques Herings* Ronald Peeters ${ }^{\dagger} \quad$ Anastas P. Tenev ${ }^{\ddagger}$ \\ Frank Thuijsman ${ }^{\S}$
}

April 10, 2019

\begin{abstract}
In a local interaction model agents situated on a circle play bilateral prisoners' dilemmas with their immediate neighbors and have three possible strategies: cooperate in all interactions (altruistic), defect in all interactions (egoistic), or cooperate with one immediate neighbor with probability $1 / 2$ (partial cooperation). After each period the agents adopt the strategy with the highest average payoff in their observed local neighborhood (naïve imitation). The absorbing states of the process are outlined and analysed. There does not exist an absorbing state in which the partially cooperative strategy coexists with any of the other strategies. The partially cooperative strategy limits the diffusion of altruistic behavior in the population. Even though clustering of altruists is generally beneficial for sustaining altruism, relatively big groups of altruists at the onset actually enable the spread of the partially cooperative strategy.
\end{abstract}

Keywords: Altruism; Public goods; Imitation; Local interaction

JEL Classification: C63, C70, C72, C73

*Department of Economics, Maastricht University, P.O. Box 616, 6200 MD Maastricht, The Netherlands. E-mail: p.herings@maastrichtuniversity.nl

${ }^{\dagger}$ Department of Economics, University of Otago, P.O. Box 56, Dunedin 9054, New Zealand. E-mail: ronald.peeters@otago.ac.nz

${ }^{\ddagger}$ Department of Economics, Maastricht University, P.O. Box 616, 6200 MD Maastricht, The Netherlands. E-mail: ap.tenev@maastrichtuniversity.nl

$\S$ Department of Data Science and Knowledge Engineering, Maastricht University, P.O. Box 616, 6200 MD Maastricht, The Netherlands. E-mail: f.thuijsman@maastrichtuniversity.nl 


\section{Introduction}

A lot of economic thought has been devoted to the factors and systems that are conducive to altruism, so that public goods can be sufficiently financed and the systems in place can be upheld efficiently and sustainably. While theoretical work has identified that altruistic behavior has slim chances of survival if agents use best responses, many results point to the fact that altruism can be sustained in a local interaction framework where the agents are imitators (Bergstrom and Stark, 1993; Eshel et al., 1998; Young, 1998; Bergstrom, 2002; Levine and Pesendorfer, 2007). A common feature of these models is that they often include two possible strategies for the agents involved: they are either cooperators or non-cooperators, i.e. altruists or egoists (Nowak and May, 1992; Ellison, 1993; Fosco and Mengel, 2011; GarcíaMartínez and Vega-Redondo, 2015). However, there is evidence that at least three general types of people can be distinguished in an organisation: givers, takers and matchers (Grant, 2013). Givers are cooperating altruists, takers are non-cooperating egoists, and matchers strive for a balance in their giving and receiving (Mäthner and Lanwehr, 2017).

Attempting to bring the models closer to observed reality, this paper adds a third possible strategy to the public goods game of Eshel et al. (1998) by allowing the agents to treat their neighbors differently, cooperating with only some of them, i.e. they exhibit partial cooperation and contribute to one of their neighbors and are egoistic to the other one. Which of the neighbors they would cooperate with depends on a probabilistic realization. This happens on a fixed simplified network structure, a circle, and using naïve imitation such that at each period agents imitate the strategy with the highest average payoff in their observed neighborhood. The range that agents immediately affect with their choices and about which they have full information with regards to chosen strategies and obtained payoffs consists of only their two direct neighbors.

The local interaction intrinsic in the model allows its interpretation as a local public goods model. Local public goods are public goods that can be enjoyed in a particular geographic area. In a broader context, this means that a selection of all agents work together towards a common goal and, if achieved, the goal would benefit everyone in the neighborhood equally. It is possible to conceptualise the local communities as clubs formed to provide local public goods (Hindriks and Myles, 2013). The concept of local public goods can be used in contexts involving various scales, from considering the interactions of different countries or states on climate control acts to very small scale interaction involving a few participants, for example, pair programming. ${ }^{1}$

It is difficult to foresee how altruism would fare in such an environment, especially if the partial cooperators have an equal chance of cooperating or not with a particular neighbor. The current paper addresses this main research question by investigating the absorbing states

\footnotetext{
${ }^{1}$ This is a way of developing software whereby two programmers work on one work station - one of them writing the code and focusing on the technical aspects of the current code, while the other one reviews the code and considers the strategic direction of the work. The two participants often change roles.
} 
of the resulting process, their frequency of occurrence, times to absorption, and initial configurations of agents' strategies that are most supportive of outcomes with a high proportion of altruist acts.

One of the main results of this paper is that the partially cooperative strategy cannot coexist with the other strategies in an absorbing state. In fact, the partially cooperative strategy hinders the spread of altruism by enabling the egoists' strategy even more than it favors its own proliferation in the population. Somewhat in contrast to other models, due to this underlying dynamic, relatively big groups of altruists at the beginning stage are beneficial for the partial cooperators, who in turn facilitate the egoists. Even though clustering of altruists is assumed to be best for cooperative outcomes, here a middle range of segregation of altruists helps the spread of altruism the most.

This paper contributes to the strand of literature on public goods in networks. Since models that rely on a best response dynamic typically produce results dominated by egoistic behavior (Young, 1998), many models have assumed boundedly rational behavior, expressed by a variety of decision rules. These span from imitating the strategy with the highest average payoff in the observed neighborhood (Eshel et al., 1998), to imitating the strategy with the highest payoff (Matros, 2012), additionally taking the linking choices of the imitated agents into account (Fosco and Mengel, 2011), etc. These behavioral decision rules imply little oversight of the agents over the game or system that they are part of and little or no knowledge of the available strategies and their potential payoffs.

To elaborate on the latter two contributions, Matros (2012) investigates whether altruism can survive when agents can choose between naïve imitation and myopic best-reply rules. He finds that altruistic behavior can be sustained if local neighborhoods are not too small or too large both in short-run and in long-run settings. Moreover, when the agents can use both decision rules, altruism survives in all cases except in those in which the decision rules are introduced into the population randomly. In a setting with naïve imitation and endogenous link formation on a more complex graph, Fosco and Mengel (2011) find that coexistence of cooperators and defectors is possible. However, it results in either their full separation or the cooperators taking a central position in the graph and the defectors being marginalised. Unlike the setting in the current model, not all agents revise their choices simultaneously at every period, but only a random sample of them do so. Moreover, their model includes different information and interaction neighborhoods and shows that the results are robust towards variations in these neighborhoods. This is in contrast to Mengel (2009), where under the framework of Eshel et al. (1998), the polymorphic states featuring altruists and egoists are not stochastically stable if the agents use information from beyond their interaction neighborhood. Mengel (2009) also finds that the co-existence result from Eshel et al. (1998) does not extend to general networks. However, introducing conformist bias (if an action is more popular, the agent is more likely to use it) stabilizes the cooperation results. Also Eshel et al. (1999) and Eshel et al. (2000) find that, even with a bigger size of the information 
neighborhood as compared to the interaction neighborhood, altruism is sustainable. In this study the finding crucially depends on a conservative learning assumption where an individual adopts a specific strategy only if it is used by at least one of his immediate neighbors.

The remainder of the paper is organised as follows. The next section describes the specifics of the model. Afterwards, the absorbing states are first outlined analytically and next investigated by means of computer simulations. The last section discusses the findings and contains the conclusion.

\section{The model}

This model extends the model of local public goods provision of Eshel et al. (1998) by adding the possibility for agents to be partially altruistic. Within this extended context it investigates and showcases the dynamics of behavior when agents follow the naïve heuristic to imitate the strategy that performed on average the best among the strategies observed within their neighborhoods.

\subsection{Local public goods game}

There are $n \geq 3$ agents on a circle and $N=\{1,2,3, \ldots, n\}$ denotes the set of agents. Every agent interacts with his two direct neighbors and exhibits either egoistic or altruistic behavior towards each of them. All agents have three possible strategies at their disposal: the set of strategies is $S=\{A, P, E\}$, where $A$ represents the altruistic strategy, $E$ represents the egoistic strategy, and $P$ represents the partially altruistic strategy.

Every two neighbors share a local public good (LPG). Altruistic contributions to the LPG benefit the contributors and the neighbor with whom they share the good but they also impose costs to the contributors. Agents who behave egoistically do not invest in a particular LPG and thus bear no costs. However, they benefit from the LPG provision if their neighbors have contributed. Altruists $(A)$ contribute to both LPGs they can support (i.e., the one they share with their left-hand and the one they share with their right-hand neighbor), while egoists contribute to neither of them. The partially altruistic strategy $(P)$ enables agents to be altruistic to only one of their neighbors. Strategy $P$ manifests itself in two possible decisions: $L$, representing altruistic behavior towards the left-hand neighbor and egoistic behavior towards the right-hand neighbor, and $R$, representing altruistic behavior towards the right-hand neighbor and egoistic behavior towards the left-hand neighbor. ${ }^{2}$ If a player uses the $P$ strategy, then in every period he chooses each of the possible decisions, $L$ or $R$, with probability $1 / 2$.

A state $x=\left(x_{1}, x_{2}, \ldots, x_{n}\right)$ is given by a profile of strategies, so for all agents $i \in N$ it holds that $x_{i} \in S$. Hence, the state space is $X=S^{n}$. The vector $d=\left(d_{1}, d_{2}, \ldots, d_{n}\right)$ specifies

\footnotetext{
${ }^{2}$ Agents on the circle are numbered in clockwise direction such that agent $i-1$ is left of agent $i \geq 2$. Due to the circular structure agent $n$ is left of agent 1 .
} 
the decisions for every player in a particular state. Given a player's strategy $x_{i} \in S$, his decision $d_{i}$ belongs to the set of decisions $D_{i}\left(x_{i}\right)$ given by:

$$
D_{i}\left(x_{i}\right)= \begin{cases}\{A\} & \text { if } x_{i}=A \\ \{L, R\} & \text { if } x_{i}=P \\ \{E\} & \text { if } x_{i}=E .\end{cases}
$$

The decision space is $D=\{A, L, R, E\}^{n}$. The feasible realizations in state $x \in X$ belong to $D(x)=\prod_{i \in N} D_{i}\left(x_{i}\right)$.

Let $\pi_{i}: D \rightarrow[-2 c, 2]$ denote the payoff function for agent $i$, where $c \in(0,1 / 4)$ is a fixed constant representing the cost of an altruist act. The function can be decomposed as $\pi_{i}(d)=b_{i}(d)-c_{i}(d)$, given that the benefits for every agent are

$$
b_{i}(d)= \begin{cases}2 & \text { if } d_{i-1} \in\{R, A\} \text { and } d_{i+1} \in\{L, A\} \\ 1 & \text { if either } d_{i-1} \in\{R, A\} \text { or } d_{i+1} \in\{L, A\} \\ 0 & \text { otherwise }\end{cases}
$$

and the costs are

$$
c_{i}(d)= \begin{cases}2 c & \text { if } d_{i}=A \\ c & \text { if } d_{i} \in\{L, R\} \\ 0 & \text { if } d_{i}=E .\end{cases}
$$

Every interaction (with left-hand or right-hand neighbor) in which the agent is altruistic costs him $c$ and it brings him a benefit of $N_{A}$, where $N_{A} \in\{0,1,2\}$ is the number of altruistic acts towards him. ${ }^{3}$ For example, if an agent uses strategy $A$ and is surrounded from the left by a decision $R$ and from the right by a decision $L$, then there are 2 altruist acts by his neighbors towards him, 1 by each of his neighbors, while he commits 2 altruist acts towards his neighbors. Therefore his payoff is $2-2 c$. Even though this payoff structure renders $E$ the sole survivor in a best response dynamic and the only rationalizable strategy, altruistic behavior can survive when a naïve imitation rule is adopted.

The partial strategy bridges the two extremes of behavior following from strategies $A$ and $E$. In reality people often prefer the middle ground and do not go for the extremes. There are many conceivable ways to define a partial strategy. Examples are the reciprocal partial strategy, which responds to a neighbor with the action he took in the previous period, or the toss of a coin every period to determine if one would assume an $A$ or $E$ strategy. The current paper's definition of the partial strategy satisfies history-independence, generates exactly one altruistic act with probability one, and treats the two neighbors symmetrically.

\footnotetext{
${ }^{3}$ The values for $c$ are within $(0,1 / 4)$ because in case $c>1 / 4$ altruism becomes too difficult to sustain and even $E$ and $A$ strategies cannot coexist. It follows logically that the more expensive altruism is, the more difficult it would be to sustain. In light of this observation, costs $c$ in the interval $(0,1 / 4)$ are the most favorable to altruism and therefore the most interesting to investigate. When $c=1 / 4$ there are payoff ties and for this reason the inequality is taken to be strict.
} 


\subsection{Naïve imitation dynamics}

The agents interact repeatedly and can reevaluate their strategies once per period. Assuming that every agent has full information about the strategies and payoffs of his direct neighbors, the actions of the agents are based on naïve imitation which is described by the following rule: Every agent considers his direct neighbors' payoffs in the current state and in the next period imitates the strategy yielding the highest average payoff from the ones he observes, including his own. This does not exclude that the agent plays his current strategy in the next period. It also implies that if an agent and all his neighbors use the same strategy the agent will continue employing this strategy. More formally, for some given agent $i \in N$ at a decision profile $d \in D$, let $N_{s}^{i}(d)=\left\{j \in\{i-1, i, i+1\} \mid d_{j} \in D_{j}(s)\right\}$ be the set of neighbors of agent $i$ (including agent $i$ himself) that employ strategy $s \in S$ and define:

$$
\mu_{s}^{i}(d)= \begin{cases}\sum_{k \in N_{s}^{i}(d)} \frac{\pi_{k}(d)}{\left|N_{s}^{i}(d)\right|} & \text { if }\left|N_{s}^{i}(d)\right| \geq 1 \\ -1 & \text { otherwise. }\end{cases}
$$

Here $\mu_{s}^{i}(d)$ gives the average observed payoff per strategy $s$ for an agent $i$ at decision profile $d$. If a strategy is not observed in the neighborhood of the agent, then, for technical reasons, it gets a value of -1 , which ensures it will not be implemented. Then the naïve imitation decision rule articulated above can be summarized as $f: D \rightarrow X^{n}$, where for $i \in N$ it holds that $f_{i}(d)=\operatorname{argmax}_{s \in S} \mu_{s}^{i}(d)$. The particular payoff functions ensure that the maximum average payoff is uniquely determined.

Next, for $x \in X$, let

$$
F(x)=\{y \in X \mid \text { there exists a } d \in D(x) \text { such that } y=f(d)\}
$$

denote the set of states that, starting from $x$, can be reached with positive probability in one iteration. In the presence of the $P$ strategy, the set $F(x)$ can contain more than one element. Further, let $F^{m}(x)$ denote the states that are reached with positive probability from $x$ in $m$ steps (with $F(x)=F^{1}(x)$ ).

In the process outlined so far, agents follow a decision rule that requires only information from the current state to fully define the next one - the crucial property for a Markovian process. Let $Q_{x y}$ denote the probability to change from state $x$ to state $y$ after one single iteration of the imitation process. It holds that $Q_{x y}>0$ if and only if $y=f(d)$ with $d \in D(x)$. The matrix of transition probabilities $\left[Q_{x y}\right]_{x, y \in X}$, along with a specification of the initial state $x^{0} \in X$, determines a Markov process on the state space $X$. A set of states $\mathcal{X}$ is absorbing if it is a minimal set of states such that once a state in the set is reached, the Markov process cannot leave this set. In other words, the system cannot transition into another minimal set of states once it has reached this particular set of states (Ross, 2000). The absorbing sets of the current imitation process will be examined in detail in Section 3. 
If every agent in a state uses the same strategy, then this produces a singleton absorbing set. Therefore the model has at least three absorbing sets, corresponding to the three singleton absorbing sets where all agents use strategies $A, P$, and $E$ respectively. When only strategies $A$ and $E$ are possible, once the $E$ strategy appears in a state it can only be isolated in small pockets but will never disappear. The $P$ strategy leads to a "rock-paper-scissors" structure and can potentially completely eliminate $E$ strategies from states. These possibilities will be explored further using simulations in Section 4.

\subsection{Preliminaries}

A number of definitions are necessary to clarify the content of the next parts.

Definition 1 (segments). For $a, b \in N$, the segment $[a, b]$ is the set of agents defined by:

$$
[a, b]= \begin{cases}\{i \in N \mid a \leq i \leq b\} & \text { if } a \leq b \\ \{i \in N \mid a \leq i \text { or } i \leq b\} & \text { if } a>b .\end{cases}
$$

Hence, a segment consists of a number of consecutive agents on the circle, irrespective of their strategies.

Definition 2 (strings). For $k \in N$ and strategy $s \in S$, a ks-string of the state $x \in X$ is a segment $[a, b]$ such that the cardinality of $[a, b]$ is equal to $k$, for every $i \in[a, b], x_{i}=s$ and if $k<n$ then $x_{a-1} \neq s$ and $x_{b+1} \neq s$.

In other words, a $k s$-string consists of a locally maximal number of consecutive agents on the circle who employ the same strategy in a particular iteration of the imitation process. Based on Definition 2, the notation $\geq m s$-string is used as shorthand for $k s$-strings with $k \geq m$. The generic name $s$-string will be used for a $k s$-string of any length.

For a state $x \in X$, let $p_{1}(x)$ be the number of $1 P$-strings of $x$ and $p_{\geq 2}(x)$ be the number of $\geq 2 P$-strings of $x$. By analogy $e_{2}(x)$ is the number of $2 E$-strings of $x, a_{\geq 2}(x)$ is the number of $\geq 2 A$-strings of $x$, etc. The notation $a(x)=a_{\geq 1}(x), e(x)=e_{\geq 1}(x)$ and $p(x)=p_{\geq 1}(x)$ is used as shorthand for the total number of $A$-strings, $E$-strings, and $P$-strings, respectively.

In the model of Eshel et al. (1998) all agents employ the strategies $A$ and $E$ and all state transitions are deterministic. The following lemma presents one of their results, where absorbing sets consisting of two states with deterministic transitions between them are called blinkers.

Lemma 1 (Eshel et al., 1998). Let $x, y \in X$ be such that, for every $i \in N, x_{i} \in\{A, E\}$ and $y \in F(x)$. The set $\{x, y\}$ is a blinker if and only if: (i) $e_{1}(x)+e_{3}(x) \geq 1$; (ii) for all $k \geq 4$, $e_{k}(x)=0$; (iii) in $x$ the length of an A-string between any two consecutive E-strings is as specified in Table 1.

If there is only one $E$-string in a blinker, then the number of $A$ 's counted is the number of $A$ 's between one end of the $E$-string and the other. Note that no $\geq 4 E$-strings can exist in 


\begin{tabular}{llll}
\hline & $(E)$ & $(E, E)$ & $(E, E, E)$ \\
\hline$(E)$ & $\geq 5 A$ & $\geq 4 A$ & $\geq 3 A$ \\
$(E, E)$ & & $\geq 3 A$ & $\geq 3 A$ \\
$(E, E, E)$ & & & $\geq 3 A$ \\
\hline
\end{tabular}

Table 1: Length of an $A$-string between $E$-strings of different lengths within blinkers. (Due to symmetry only half of the table is filled.)

a blinker. Blinkers can only occur when $n \geq 6$. An example of a blinker is presented in the next subsection.

\subsection{Two detailed examples}

This section provides two examples. The first one constitutes a blinker and the second one illustrates how, given the partial strategy, starting from a specific state more than one absorbing state can be reached.

First, consider the situation with $n=6$ agents with the state being $\left(x_{1}, x_{2}, x_{3}, x_{4}, x_{5}, x_{6}\right)=$ $(A, A, E, A, A, A)$. Consider $y \in F(x)$. Since agent 1 adopts the $A$ strategy and is surrounded by neighbors also adopting the $A$ strategy, he does not experience the use of any other strategy and will continue to play the $A$ strategy: $y_{1}=A$. The same applies to agents 5 and 6: $y_{5}=y_{6}=A$. Both agents 2 and 4 receive a payoff of $1-2 c$ and observe one $A$ playing neighbor who receives a payoff of $2-2 c$ and one $E$ playing neighbor who receives a payoff of 2 . On average, they find the $E$ strategy to be more rewarding than the $A$ strategy $(2>3 / 2-2 c)$ and both shift to the $E$ strategy: $y_{2}=y_{4}=E$. Agent 3 sticks to the $E$ strategy as it yields a better payoff than the $A$ strategies of his neighbors $(2>1-2 c): y_{3}=E$. Hence, as also illustrated in Table 2 , after one iteration the state changes to $\left(y_{1}, y_{2}, y_{3}, y_{4}, y_{5}, y_{6}\right)=$ $(A, E, E, E, A, A)$. From here, agents 3 and 6 stick to their strategies as they do not observe any other strategy: $z_{3}=E$ and $z_{6}=A$. Agents 1 and 5 stick to the $A$ strategy, since $((1-2 c)+(2-2 c)) / 2=3 / 2-2 c>1: z \in F(y)$ satisfies $z_{1}=z_{5}=A$. It is already clear that the $E$ strategy does not spread further, this being a consequence of the boundary $A$ playing agents observing an $A$ player that is fully surrounded by $A$ players. This underlines the importance of having a sufficiently large string of $A$ players in between the $E$-strings (Table 1). Finally, agents 2 and 4 both observe agent 3 being very unsuccessful with the $E$ strategy such that on average the $A$ strategy they observe is more productive $((1+0) / 2=1 / 2<1-2 c)$ and this leads them back to it: $z_{2}=z_{4}=A$. The process is back at the starting state: $\left(z_{1}, z_{2}, z_{3}, z_{4}, z_{5}, z_{6}\right)=(A, A, E, A, A, A)=\left(x_{1}, x_{2}, x_{3}, x_{4}, x_{5}, x_{6}\right)$.

Second, consider the situation with $n=4$ agents with the state being $\left(x_{1}, x_{2}, x_{3}, x_{4}\right)=$ $(A, P, P, E)$. In this state there are four possible realizations for the pair of $P$ strategies: $d$ equals $(A, R, R, E),(A, L, L, E),(A, L, R, E)$ or $(A, R, L, E)$, each with equal probability. These realizations induce a transition to the next state being $(E, P, E, E),(P, P, E, E)$, 


\begin{tabular}{lll}
\hline Current state $(x)$ & Payoffs in current state $(\pi(d))$ & Next state $(y)$ \\
\hline$(A, A, E, A, A, A)$ & $(2-2 c, 1-2 c, 2,1-2 c, 2-2 c, 2-2 c)$ & $(A, E, E, E, A, A)$ \\
$(A, E, E, E, A, A)$ & $(1-2 c, 1,0,1,1-2 c, 2-2 c)$ & $(A, A, E, A, A, A)$ \\
\hline
\end{tabular}

Table 2: Example of a blinker. The two segments of the table comprise a blinker for the smallest possible circle length for which blinkers can exist, $n=6$.

( $E, A, E, E)$ or $(P, P, P, E)$, respectively; again, each with equal probability. While from states $(E, P, E, E)$ and $(E, A, E, E)$ the process moves towards the absorbing state $(E, E, E, E)$, from the other two states further continuation of the process again depends on the realizations of the $P$ strategies. Table 3 presents the states that can be visited in the process starting from state $x$. Figure 1 illustrates the full process of state transitions and shows that, in each of these states the probability to reach the absorbing state $(P, P, P, P)$ equals $1 / 4$, and that in case the process does not move to this state it will ultimately end up in the egoistic absorbing state. In short, given that the process starts in state $x$, it evolves to the absorbing state $(E, E, E, E)$ with probability $7 / 8$ and to the absorbing state $(P, P, P, P)$ with probability $1 / 8$. This illustrates the possibility for the process to reach different absorbing states with different payoff levels: in $(P, P, P, P)$ the average payoff is $1-c$, while it equals 0 in $(E, E, E, E)$.

\begin{tabular}{lllll}
\hline Current state & Prob. & Current decisions & Current payoffs & Next state \\
\hline$(A, P, P, E)$ & $1 / 4$ & $(A, R, R, E)$ & $(-2 c, 1-c, 1-c, 2)$ & $(E, P, E, E)^{a}$ \\
& $1 / 4$ & $(A, L, L, E)$ & $(1-2 c, 2-c,-c, 1)$ & $(P, P, E, E)$ \\
& $1 / 4$ & $(A, L, R, E)$ & $(1-2 c, 1-c,-c, 2)$ & $(E, A, E, E)^{a}$ \\
$(P, P, E, E)$ & $1 / 4$ & $(A, R, L, E)$ & $(-2 c, 2-c, 1-c, 1)$ & $(P, P, P, E)$ \\
& $1 / 4$ & $(R, R, E, E)$ & $(-c, 1-c, 1,0)$ & $(P, E, P, E)^{a}$ \\
& $1 / 4$ & $(L, L, E, E)$ & $(1-c,-c, 0,1)$ & $(E, P, E, P)^{a}$ \\
& $1 / 4$ & $(L, R, E, E)$ & $(-c,-c, 1,1)$ & $(E, E, E, E)$ \\
$(P, P, P, E)$ & $1 / 4$ & $(R, L, E, E)$ & $(1-c, 1-c, 0,0)$ & $(P, P, P, P)$ \\
& $1 / 8$ & $(L, L, L, E)$ & $(1-c, 1-c,-c, 1)$ & $(E, P, E, E)^{a}$ \\
& $1 / 8$ & $(L, L, R, E)$ & $(1-c,-c,-c, 2)$ & $(E, P, E, E)^{a}$ \\
& $1 / 8$ & $(L, R, L, E)$ & $(-c, 1-c, 1-c, 1)$ & $(E, P, E, E)^{a}$ \\
& $1 / 8$ & $(R, L, L, E)$ & $(1-c, 2-c,-c, 0)$ & $(P, P, P, P)$ \\
& $1 / 8$ & $(L, R, R, E)$ & $(-c,-c, 1-c, 2)$ & $(E, P, E, E)^{a}$ \\
& $1 / 8$ & $(R, R, L, E)$ & $(-c, 2-c, 1-c, 0)$ & $(P, P, P, P)^{a}$ \\
& $1 / 8$ & $(R, L, R, E)$ & $(1-c, 1-c,-c, 1)$ & $(E, P, E, E)^{a}$ \\
& $1 / 8$ & $(R, R, R, E)$ & $(-c, 1-c, 1-c, 1)$ & $(E, P, E, E)^{a}$ \\
\hline
\end{tabular}

Table 3: Example of the development of the process. It reaches two types of absorbing states, given a specific starting state. The states marked with an ${ }^{a}$ reach absorption in the following period with probability 1 . 


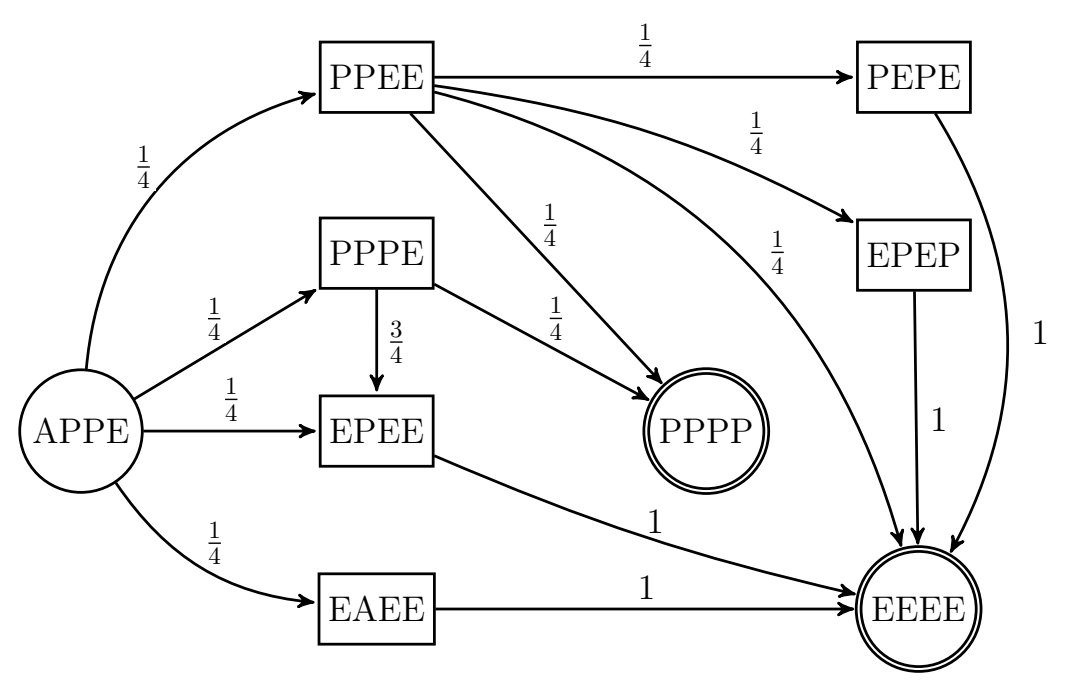

Figure 1: Example of the development of the imitation dynamics starting from a state $(A, P, P, E)$ in the circle on the left and reaching one of two possible absorbing sets (in double circles) within 2 or 3 iterations. The transition probabilities between the different states are given above the arrows pointing in the direction of the transitions.

\section{Theoretical results}

This section describes the different absorbing sets that the process can reach. There are two general types of absorbing sets that will be considered separately: stationary states (singleton absorbing sets) and non-singleton absorbing sets. After characterizing the two types of absorbing sets, the section continues with some further analytical observations.

Proposition 1. A state $x \in X$ is a stationary state if and only if: (i) all agents play the same strategy; or (ii) $a_{\leq 2}(x)=0, a_{\geq 3}(x) \geq 1, e_{1}(x)=0, e_{2}(x) \geq 1, e_{\geq 3}(x)=0$ and $p(x)=0$.

Proof. Eshel et al. (1998) demonstrate that states consisting of only $A$ 's, only $E$ 's or states which consist of $2 E$-strings and $\geq 3 A$-strings are stationary. Introducing the $P$ strategy does not affect this result. What is left to prove is that in the case of three possible strategies for the agents, the only additional stationary state is the one where all agents play the strategy $P$. In this case all agents are surrounded by the same strategy $P$ and no agent will change his strategy according to the naïve imitation rule. Hence a state with only $P$ strategies is also a stationary state. It remains to be shown that there are no stationary states in which some but not all players use strategy $P$.

Let $x \in X$ be a state such that $x_{i}=P$ for some $i \in N$ and at least one of his immediate neighbors uses strategy $A$ or $E$. Let $y \in F(x)$ be a state reached from $x$ in one iteration.

Case 3.1: $\left(x_{1}, x_{2}\right)=(E, P)$

Take $d \in D(x)$ such that $d_{2}=L$. It follows that $\pi_{1}(d) \geq 1$ and $\pi_{2}(d) \in\{-c, 1-c\}$. There are three possibilities for the strategies of the third agent: (i) $x_{3}=E: \pi_{2}(d)=-c$ and $\pi_{3}(d) \geq 0$. 
(ii) $x_{3}=P: \pi_{3}(d) \leq 1-c$. (iii) $x_{3}=A: \pi_{3}(d) \leq 1-2 c$. For all possibilities it holds that $y_{2}=E \neq x_{2}$.

Case 3.2: $\left(x_{1}, x_{2}\right)=(P, A)$

For every $d \in D(x), \pi_{1}(d) \geq 1-c$. Two subcases can be distinguished:

(i) $x_{3} \in\{E, P\}$ : If $d_{1}=L$, then $\pi_{2}(d) \leq 1-2 c$ and $\pi_{3}(d) \geq 1-c$, so $x_{2} \neq y_{2} \in\{E, P\}$.

(ii) $x_{3}=A$ : In case $n=3, y=(P, P, P)$ for every $d \in D(x)$. Next consider $n \geq 4$. Two cases are distinguished, since if $d_{0}=E$ this is identical to Case 3.1(iii).

(a) $d_{0}=L$ : Take $d_{1}=R$, then $\pi_{0}(d), \pi_{1}(d) \leq 1-c, \pi_{2}(d)=2-2 c$ and $y_{1}=A \neq x_{1}$.

(b) $d_{0} \in\{R, A\}$. It holds that $\pi_{1}(d)=2-c, \pi_{2}(d), \pi_{3}(d) \leq 2-2 c$ and $y_{2}=P \neq x_{2}$.

Therefore, with positive probability $d \in D(x)$ is such that $y(d) \neq x$, so $x$ is not a stationary state.

To approach the non-singleton absorbing sets with the right tools, some terminology is introduced in Definitions 3-8 below. The examples from the previous section will be used to illustrate these definitions afterwards.

Definition 3 (splitting). For $k \in\{1,2, \ldots, n-1\}, s \in\{A, P, E\}$ and a state $x \in X$ that transitions into a state $y \in F(x)$, a ks-string $[a, b]$ of $x$ splits if at least one of the following three conditions holds:

(i) for $k \geq 2$, at least one of the following two conditions holds:

(a) $y_{b-1}=y_{b+1}=s$ and $y_{b} \neq s$;

(b) $y_{a-1}=y_{a+1}=s$ and $y_{a} \neq s$;

(ii) for $k=2, y_{a-1}=y_{b+1}=s, y_{a} \neq s$ and $y_{b} \neq s$;

(iii) for $k=1, y_{a-1}=y_{a+1}=s$ and $y_{a} \neq s$.

Definition 4 (growing and shrinking).

(i) For $k \in\{1,2, \ldots, n-1\}, s \in\{A, P, E\}$ and a state $x \in X$ that transitions into a state $y \in F(x)$, a ks-string $[a, b]$ of $x$ grows if for every $i \in[a, b], y_{i}=s$ and $\left(y_{a-1}=s\right.$ or $\left.y_{b+1}=s\right)$.

(ii) For $k \in\{2,3, \ldots, n-1\}, s \in\{A, P, E\}$ and a state $x \in X$ that transitions into a state $y \in F(x)$, a ks-string $[a, b]$ of $x$ shrinks if $y_{a-1} \neq s, y_{b+1} \neq s$ and $\left(y_{a} \neq s\right.$ or $\left.y_{b} \neq s\right)$.

Remark: Under the imitation process, if the whole circle is one $n s$-string it can neither grow any further, nor can it shrink, because no agent will change his strategy. 
Definition 5 (disappearing). For $k \in\{1,2\}, s \in\{A, P, E\}$ and a state $x \in X$ that transitions into a state $y \in F(x)$, a ks-string $[a, b]$ of $x$ disappears if for every $i \in[a, b], y_{i} \neq s$ and: (i) if $y_{a-1}=s$, then $y_{a-2}=s$ or (ii) if $y_{b+1}=s$, then $y_{b+2}=s$.

It is worth noting that if in Definition $5 y_{a-1} \neq s$ and $y_{b+1} \neq s$, then for every $i \in[a-1, b+1]$, $y_{i} \neq s$.

The example with four agents in the previous section can illustrate the concepts that have been introduced so far. For instance, between $(A, P, P, E)$ and $(E, P, E, E)$ the $2 P$-string shrinks in length to a $1 P$-string; the $1 E$-string grows in length to a $3 E$-string; the $1 A$-string disappears.

To analyze the absorbing sets of the imitation process, it is convenient to introduce a pre-order on the set of states $X$. This motivates the following definition of improvement.

Definition 6 (improvement). For $x, y \in X$, state $y$ improves upon $x$, denoted by $y \succ x$, if one of the following two conditions holds:

(i) $p(y)<p(x)$

(ii) $p(y)=p(x)$ and $\left[p_{n}(y), p_{n-1}(y), \ldots, p_{1}(y)\right]>_{l e x}\left[p_{n}(x), p_{n-1}(x), \ldots, p_{1}(x)\right]$.

In other words, a state $y \in X$ is an improvement upon a state $x \in X$ if it has a smaller overall number of $P$-strings or, if it has the same number of $P$-strings, then it has more longer $P$ strings in a lexicographic sense. This is considered an improvement because it implies that the state $y$ is closer to an absorbing state with only $P$ 's or to an absorbing state with no $P$ 's. Decisions of agents within a $P$-string which cause a specific $P$-string not to split will be referred to as "no-splitting decisions". These no-splitting decisions are provided explicitly by means of Definition 7.

Definition 7 (no splitting). Take a state $x \in X$ and $i \in N$. The no-splitting decision $d_{i}^{*}$ of agent $i$ at $x$ is defined by:

(i) if $i$ belongs to a $1 P$-string, then $d_{i}^{*}=R$ if $x_{i-1}=E$ and $x_{i+1}=A$, and $d_{i}^{*}=L$ otherwise;

(ii) if $i$ belongs to $a \geq 2 P$-string $[a, b]$, then $d_{i}^{*}=R$ if $i \in\{a, b-1\}$, and $d_{i}^{*}=L$ otherwise;

(iii) if $x_{i} \in\{A, E\}$, then $d_{i}^{*}$ is trivially defined as $d_{i}^{*}=x_{i}$.

In other words, the no-splitting decisions for a $2 P$-string are $\left(d_{1}^{*}, d_{2}^{*}\right)=(R, L)$; for $3 P$-strings they are $\left(d_{1}^{*}, d_{2}^{*}, d_{3}^{*}\right)=(R, R, L)$, for $4 P$-strings they are $\left(d_{1}^{*}, d_{2}^{*}, d_{3}^{*}, d_{4}^{*}\right)=(R, L, R, L)$, etc. The next lemma states that the no-splitting decisions indeed result in no splitting of a specific $P$-string.

Lemma 2. Let $[a, a+k-1]$ be a kP-string of the state $x \in X, d \in D(x)$ be such that, for every $i \in[a, a+k-1], d_{i}=d_{i}^{*}$ and $y=f(d)$. Then $[a, a+k-1]$ does not split. 
Proof. See Appendix.

If all agents take the no-splitting decision, then the number of $P$-strings does not increase in the next iteration of the imitation process. Moreover, if a particular $P$-string disappears whereas all agents outside the $P$-string take the no-splitting decision, then the number of $P$-string has gone down in this iteration of the imitation process. To formalize this, the additional notion of a $P$-section is needed.

Definition 8 (P-section). For $x \in X$, let $\mathcal{P}(x)=\left\{\left[a_{1}, b_{1}\right],\left[a_{2}, b_{2}\right], \ldots,\left[a_{r}, b_{r}\right]\right\}$ be the collection of P-strings of $x$ with $a_{1}<a_{2}<\ldots<a_{r}$ and $r=p(x)$. The set $J \subseteq N$ is called a P-section of size $k \in\{1, \ldots, p(x)\}$ of state $x$ if there is $q \in\{1, \ldots, r\}$ such that $J=\left[a_{q}-1, b_{q+k-1}+1\right]$.

Finally, for $x \in X, p_{[a, b]}(x)$ is used to denote the number of $P$-strings intersecting the interval $[a, b]$.

Lemma 3 states that if all agents in a $P$-section take the no-splitting decision, then the number of $P$-strings in this section will not go up.

Lemma 3. Let $x \in X$ and $J$ be a P-section of size $k$ of the state $x$. Let $d \in D(x)$ be such that, for every $j \in J, d_{j}=d_{j}^{*}$. Then for $y=f(d)$ it holds that $p_{J}(y) \leq p_{J}(x)=k$. Moreover, if $k=p(x)-1$ and the $P$-string in $N \backslash J$ disappears, then $p(y) \leq p(x)-1$.

Proof. By Lemma 2 it holds that none of the $P$-strings in $J$ splits. The definition of splitting implies that if $[a, b] \subset J$ is a $P$-string of state $x$, then $y$ has at most one $P$-string intersecting the interval $[a-1, b+1]$. It follows that $p_{J}(y) \leq p_{J}(x)$.

Assume $k=p(x)-1$ and the $P$-string in $N \backslash J$, say $\left[a_{q}, b_{q}\right]$, disappears. The result follows immediately if $y_{a_{q}-1} \neq P$ and $y_{b_{q}+1} \neq P$. If $y_{a_{q}-1}=P$, then by the definition of disappearing it holds that $y_{a_{q}-2}=P$. Since $\left[a_{q-1}, b_{q-1}\right]$ does not split, it follows that $a_{q}-1$ and $a_{q}-2$ are part of the unique $P$-string intersecting $\left[a_{q-1}-1, b_{q-1}+1\right]$. It follows that $p(y)=p_{J}(y) \leq p_{J}(x)=p(x)-1$. A symmetric argument applies if $y_{b_{q}+1}=P$.

The inequalities in Lemma 3 are strict if a $P$-string in $J$ disappears or if two or more $P$-strings in $J$ merge.

The next lemma makes clear that at states where some but not all agents use strategy $P$, it is always possible to find an improvement in a finite number of iterations.

Lemma 4. Let $k \in\{1, \ldots, n-1\}$ be the cardinality of the longest P-string of state $x \in X$. Then there is $m \in\{1, \ldots,\lfloor k / 2\rfloor+2\}$ and $y \in F^{m}(x)$ such that $y \succ x$.

Proof. Let $[a, b]$ be any of the longest $P$-strings of $x$.

If $k=1$ and $x_{a-2}, x_{a+2} \in\{A, E\}$, then it holds by Lemma 5 in Appendix A.2 that there is $y \in F(x)$ such that $p(y) \leq p(x)$ and $y$ contains a $2 P$-string or there is $y \in F(x) \cup F^{2}(x)$ 
such that $p(y) \leq p(x)-1$. Since $[a, b]$ is one of the longest $P$-strings of $x$, it follows in all cases that $y \succ x$.

If $k=1$ and $x_{a-2}=P$ or $x_{a+2}=P$, then it holds by Lemma 6 in Appendix A.2 that there $y \in F(x) \cup F^{2}(x)$ such that $p(y) \leq p(x)$ and $y$ contains a $\geq 2 P$-string or there is $y \in F(x) \cup F^{2}(x) \cup F^{3}(x)$ such that $p(y) \leq p(x)-1$. Since $[a, b]$ is one of the longest $P$-strings of $x$, it follows in all cases that $y \succ x$.

If $k=2$, then it holds by Lemma 7 in Appendix A.2 that there is $y \in F(x) \cup F^{2}(x)$ such that $p(y) \leq p(x)$ and $y$ contains a $\geq 3 P$-string or there is $y \in F(x)$ such that $p(y) \leq p(x)-1$. Since $[a, b]$ is one of the longest $P$-strings of $x$, it follows in all cases that $y \succ x$.

If $k=3$, then it holds by Lemma 8 in Appendix A.2 that there is $y \in F(x) \cup F^{2}(x)$ such that $p(y) \leq p(x)$ and $y$ contains a $\geq 4 P$-string or there is $y \in F^{2}(x) \cup F^{3}(x)$ such that $p(y) \leq p(x)-1$. Since $[a, b]$ is one of the longest $P$-strings of $x$, it follows in all cases that $y \succ x$.

If $k \geq 4$, then it holds by Lemma 9 in Appendix A.2 that there is $y \in F(x) \cup F^{2}(x) \cup$ $F^{3}(x)$ such that $p(y) \leq p(x)$ and $y$ contains a $\geq(k+1) P$-string or $k$ is even and there is $y \in F^{k / 2}(x) \cup F^{k / 2+1}(x) \cup F^{k / 2+2}(x)$ such that $p(y) \leq p(x)-1$ or $k$ is odd and there is $y \in F^{(k+1) / 2}(x) \cup F^{(k+3) / 2}(x)$ such that $p(y) \leq p(x)-1$. Since $[a, b]$ is one of the longest $P$-strings of $x$, it follows in all cases that $y \succ x$.

The proofs of these lemmas involve constructing explicit paths to improvement for every possible case; that is, all $P$-strings of the initial state are traced to eventual disappearance or growth (also through merging with other $P$-strings) so that after a number of iterations the state has either only $P$ 's or no $P$ 's at all. Proposition 2 then concludes that the only non-singleton absorbing sets of the model are the ones in Lemma 1.

Proposition 2. A set of states is a non-singleton absorbing set if and only if it is a blinker as presented in Lemma 1.

Proof. Let $x \in X$ be any state which contains the strategy $P$ and at least one other strategy. By Lemma 4 there exists $m^{1} \in \mathbb{N}$ and $y \in F^{m^{1}}(x)$ such that $y \succ x$. Either $y$ contains no $P$ 's at all, after which the results follow from Eshel et al. (1998), or $y$ is a stationary state with only $P$ 's (Proposition 1), or we can apply Lemma 4 again and there exists $m^{2} \in \mathbb{N}$ and $z \in F^{m^{2}}(y)$ such that $z \succ y$. Since the set of states is finite and $\succ$ is a pre-order, we reach a state that contains no $P$ 's at all or a stationary state with only $P$ 's in a finite number of steps.

The section concludes with a few analytical results, complementing the simulations in the following section. The aim of these results is to show the relative advantage some strategies have over others under specific circumstances. The first series of observations deals with three specific structures of states, consisting of only $1 s$-strings, for $s \in\{A, P, E\}$ : 
(i) Let $n \geq 4$ be even and let $x$ be such that all odd agents take the same strategy $A$ and all even agents take strategy $P$. Then, all strategy $A$ agents will adopt strategy $P$ and all strategy $P$ agents will continue playing $P$.

(ii) Let $n \geq 4$ be even and let $x$ be such that all odd agents take the same strategy $E$ and all even agents take strategy $P$. Then, all strategy $P$ agents will adopt strategy $E$ and all strategy $E$ agents will continue playing $E$.

(iii) Let $n \geq 3$ be divisible by three and let $x$ be such that players alternately play $A, P$ and $E$ (i.e., $x=\cdots A P E A P E \cdots$ ). Then, all agents will adopt strategy $E$ in the next period.

The second series of observations deals with longer strings and concerns what happens at the borders of these strings, via two cases: one where a $P$-string meets an $A$-string (Case 3.3 ) and one where a $P$-string meets an $E$-string (Case 3.4 ).

Case 3.3: $n \geq 4$ and $\left(x_{1}, x_{2}, x_{3}, x_{4}\right)=(P, P, A, A)$

Let $d \in D(x)$ and $y=f(d)$. If $\left(d_{1}, d_{2}\right)=(R, R)$, then $\left(y_{2}, y_{3}\right)=(A, P)$. If $\left(d_{1}, d_{2}\right)=(R, L)$, then $\left(y_{2}, y_{3}\right)=(P, P)$. If $\left(d_{1}, d_{2}\right)=(L, R)$, then $\left(y_{2}, y_{3}\right)=(A, A)$. If $\left(d_{1}, d_{2}\right)=(L, L)$, then $\left(y_{2}, y_{3}\right) \in\{(P, A),(P, P)\}$.

Case 3.4: $n \geq 4$ and $\left(x_{1}, x_{2}, x_{3}, x_{4}\right)=(P, P, E, E)$

Let $d \in D(x)$ and $y=f(d)$. If $\left(d_{1}, d_{2}\right)=(R, R)$, then $\left(y_{2}, y_{3}\right) \in\{(E, P),(E, E)\}$. If $\left(d_{1}, d_{2}\right)=$ $(R, L)$, then $\left(y_{2}, y_{3}\right)=(P, P)$. If $\left(d_{1}, d_{2}\right)=(L, R)$, then $\left(y_{2}, y_{3}\right)=(E, E)$. If $\left(d_{1}, d_{2}\right)=(L, L)$, then $\left(y_{2}, y_{3}\right)=(P, E)$.

While this is certainly not an exhaustive list of all possibilities, some trends can be observed, such as: when $A$ 's are pitted against the $P$ 's the $P$ strategy is more likely to propagate ((i) and Case 3.3). In contrast, when $P$ 's are facing $E$ 's, the $E$ 's are more likely to take over ((ii) and Case 3.4). Finally, Eshel et al. (1998) show that many clustered $A$ 's can limit the E's to small pockets of length 2 on average. This gives a general "rock-paper-scissors" overarching structure of the setup in which any of the strategies can be defeated by one of the other strategies. This broad observation proves to be important when analysing the computer simulations in the next section.

\section{Simulation results}

This section presents the results from the computer simulations of the model. Several characteristics of the initial states have been investigated to establish which of them influence the probability of a particular absorbing set being reached. Every simulation run started with selecting an initial state based on specific parameters, including the length of the circle $n$, the probability of using the $P$ strategy and the probability of segregation for the strategies. 
Next, the decision rule was implemented until absorption was reached. For every choice of parameters the model has been simulated 10,000 times. The graphs in this section plot the averages of the specific outcome variables over these 10,000 runs. The situation where initially each agent plays one of the three strategies by uniform random choice is used as default and applies unless otherwise stated.

\section{1 $\quad$ Varying the probability the agents start with the $P$ strategy}

Let $\rho$ denote the probability by which agents use strategy $P$ in the starting state. In the default case this probability is $1 / 3$. In the extreme case when $\rho=0$ the setup is equivalent to the model by Eshel et al. (1998) and only $A$ and $E$ strategies appear in the starting state. Four other intermediate options are considered: $\rho \in\{1 / 6,1 / 2,2 / 3,5 / 6\}$. In all cases the probabilities for $A$ and $E$ in the initial state were kept equal to each other.

Absorbing sets Figure 2 presents the frequencies with which every possible absorbing set is reached. It is immediately obvious that the probability that an only $A$ stationary state appears decreases sharply when $n$ increases (top-left graph). This is due to the fact that, apart from starting at this absorbing state, it can only be reached if first all $E$ 's disappear after which the

remaining $P$ 's are driven out by the $A$ 's. However, the probability for this development is very low because strategy $A$ does worse on average when pitted against strategy $P$. The percentage of $A / E$ absorbing sets increases steadily as $n$ increases for any value of $\rho$ (top-right graph). This happens at the expense of the $E$ stationary states, which follow the opposite pattern. The $A / E$ absorbing sets are negatively affected by an increased presence of the $P$ strategy, while the $E$ 's benefit from that (bottom-left graph). At first glance it is unexpected that the $P$ stationary state is not positively influenced by more $P$ 's in the initial state (bottom-right graph). However, this can be explained by them being taken over by $E$ 's. It is interesting to note that increasing $\rho$ also increases the probability of ending in a $P$ stationary state only for small $n$, while for bigger $n$ increasing $\rho$ works in the same way only up to about $\rho=1 / 2$. After that, increasing $\rho$ further clearly decreases the probability of ending in a $P$ absorbing state, which is consistent with the explanation above. On a bigger circle, the chances that some $E$-string succeeds overturning the $P$ 's get bigger.

Times to absorption Figure 3 presents the absorption times for the process in general and conditional on a specific absorbing set being reached. Even the sets which take most iterations to reach do not require a number of iterations that exceeds 2.5 times the length of the circle. The disparate patterns of the absorption times are due to the various ways in which different absorbing states are generally reached. To get to an $A / E$ set, all $P$ 's have to disappear. While in general the $P$ 's could help eliminate all $E$ strategies, leaving even one $E$ before the $P$ 's themselves are removed suffices to reach an $A / E$ set. Absorbing to a state containing only $P$ 's always takes the most iterations, because $P$-strings can fluctuate between 

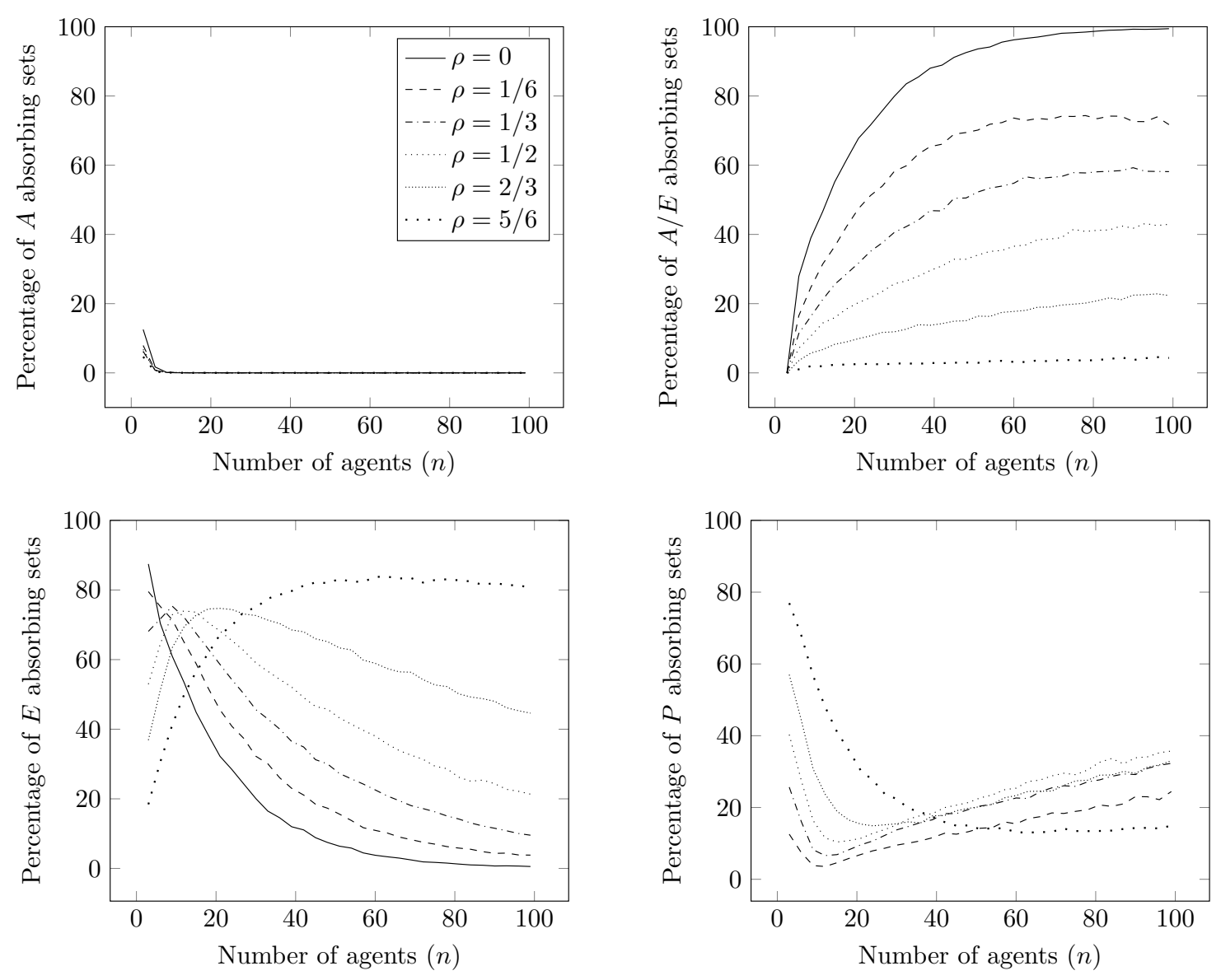

Figure 2: Percentages of specific absorbing sets resulting for different numbers of agents, varying the probability of agents starting with the $P$ strategy.

growing and shrinking much longer when surrounded by $A$ - or $E$-strings than strings of any of the two other strategies can when pitted against each other. Figure 3 does not feature absorbing times to $A$ states because they are increasingly rare as an occurrence and therefore susceptible to greater influence of outlier absorption times (see top-left graph of Figure 2).

It is noteworthy that even for higher values of $\rho$ the average absorption times are almost linear. Having more $P$ 's at the outset causes absorption to $A / E$ states to take increasingly longer (top-right), while the opposite effect is observed in the absorption to the $E$ stationary state (bottom-left). ${ }^{4}$ This is consistent with $P$ 's impeding the $A$ 's from spreading fast, while being conducive to the spread of E's.

\footnotetext{
${ }^{4}$ The flat line for $\rho=0$ in the graph for absorption to $E$ states (bottom-left) is due to the fact that when there are no $P$ 's, the setup is very similar to the neat example in the previous section and this explains the short absorption times of about 1-2 iterations. The line corresponding to the $\rho=1 / 6$ in the same graph behaves somewhat erratically at first glance. However, this is due to the fact that there are very few observations in this range.
} 

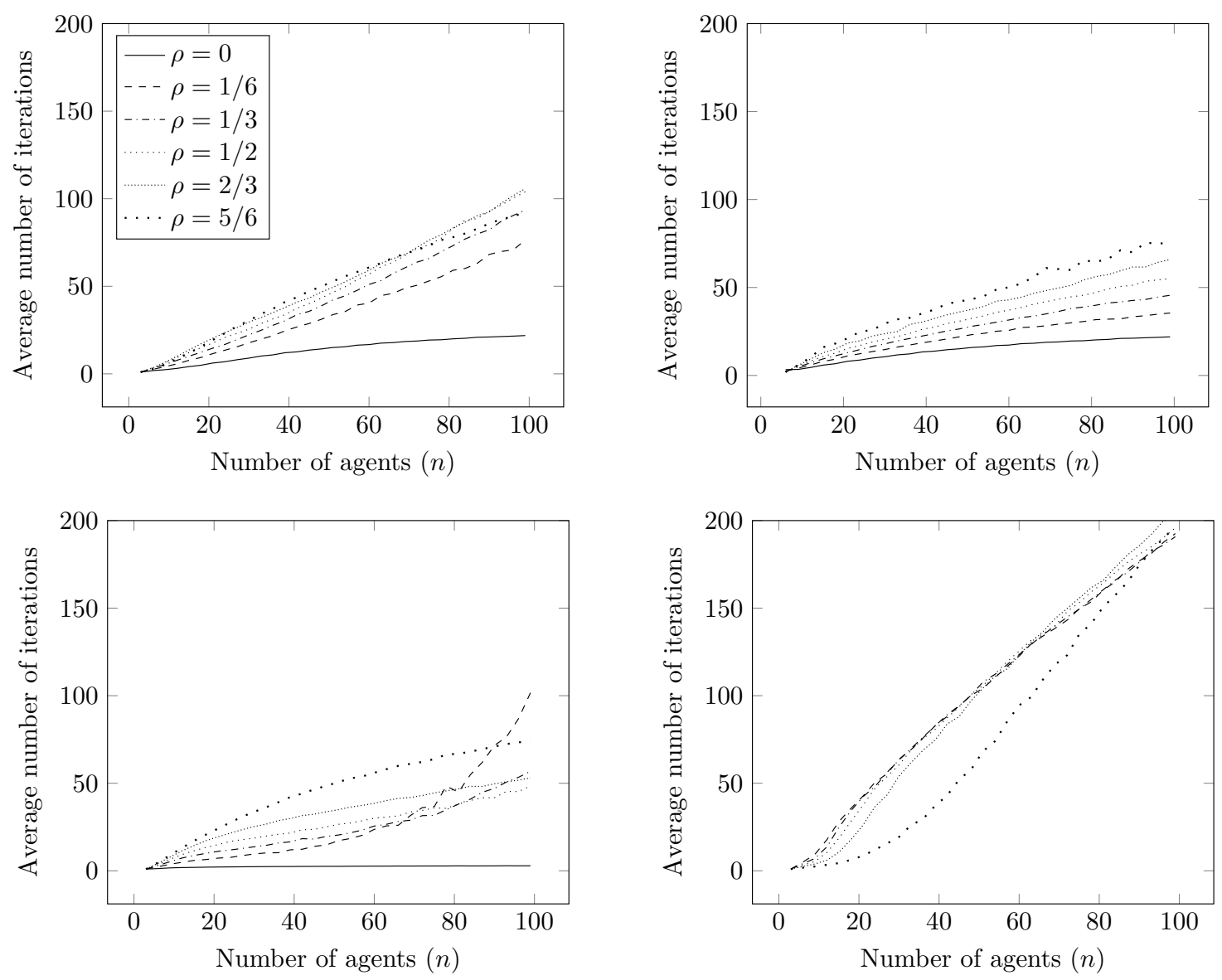

Figure 3: Average number of iterations to absorption to any absorbing state (top-left), to $A / E$ states (top-right), to $E$ states (bottom-left), and to $P$ states (bottom-right).

Strategies in the absorbing sets Figure 4 presents the collapsed results from Figure 2 divided by the three strategies rather than by absorbing sets. The results for the $A$-strategy are quite intuitive because, given the relative advantage that the $P$ strategy has over the $A$ strategy, increasing the overall number of $P$ 's in the initial state naturally leads to fewer $A$ 's in the absorbing states. Nevertheless, a circle with a higher number of agents always increases the probability that the altruists will increase in number in the absorbing states. The reverse of this is apparent for the $P$-strategy. The initially surprising dip as the number of $P$ 's in the initial states increases can be explained by the fact that small numbers of $P$ strategies, even if densely distributed, can easily be overpowered by neighboring E's. This explanation is corroborated by the data presented for the $E$-strategy, showing that $E$ 's thrive when the initial number of $P$ 's increases. The downward trend in the number of $E$ 's in absorbing states as $n$ increases can therefore be explained by a prevalence of the effect of increasing the number of agents. This is due to the fact that on a larger circle a string of $E$ 's has a higher chance of colliding with a $\geq 3 A$-string, in which case the $E$ 's inevitably shrink. 

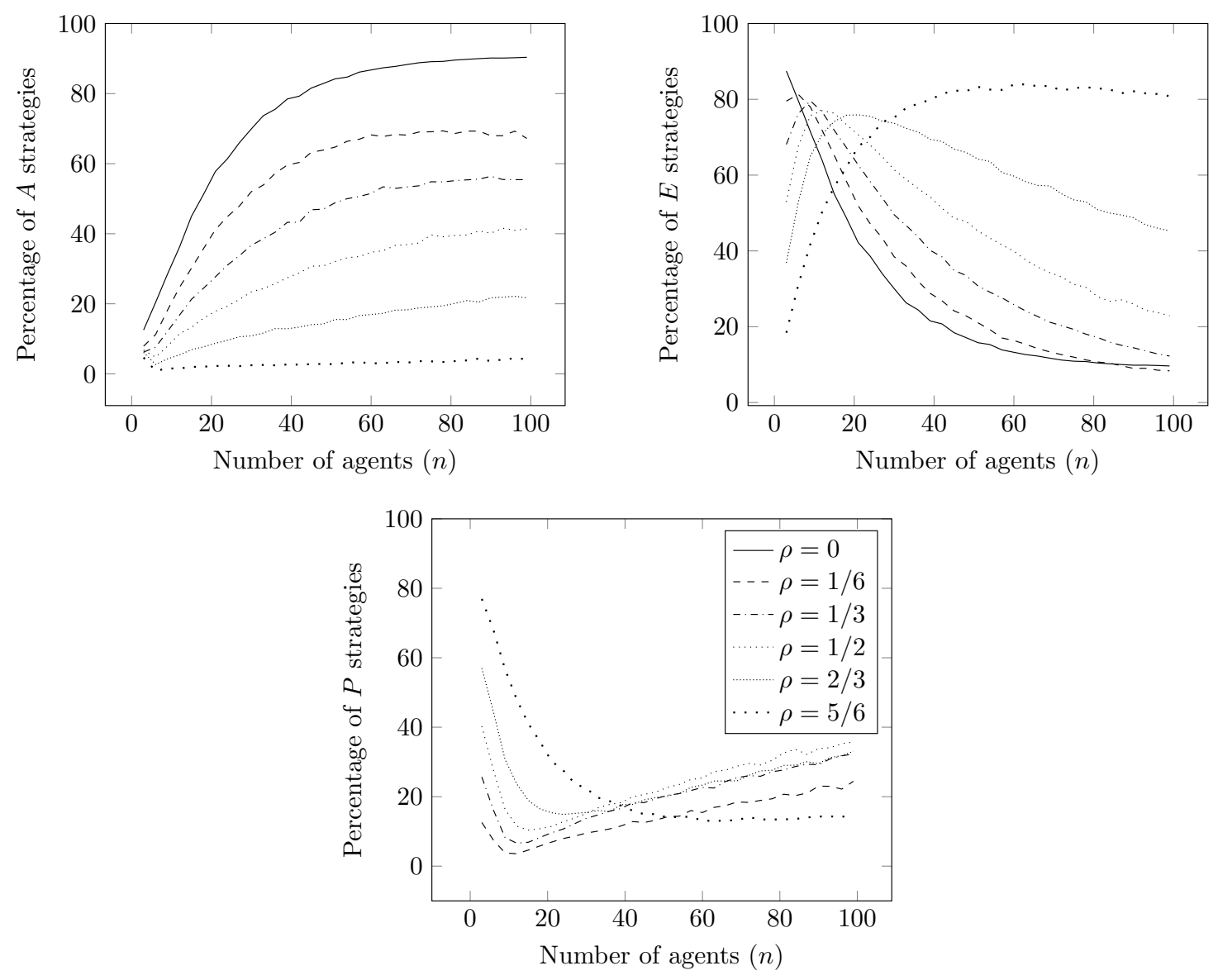

Figure 4: Percentages of specific strategies in the absorbing states resulting for different numbers of agents, varying the probability of agents starting with the $P$ strategy.

Efficiency The left graph in Figure 5 presents the average numbers of altruist acts as a proportion of the possible maximum number of altruist acts in the absorbing states. Each agent can employ two altruistic acts: one for each of the two neighbors. Strategy $A$ counts for two altruist acts, strategy $P$ for one, and strategy $E$ for zero. Since this number reflects the total value that is created in absorbing states, it measures efficiency.

Within the current setup the average percentage of altruist acts in the starting state equals $50 \%$ for all values of $\rho$. The graph shows that while efficiency improves with a bigger number of agents on the circle, the increased prevalence of the $P$ strategy in the starting state actually counters this effect. However, in states in which $A$ and $E$ coexist, $P$ marginally decreases the number of free-riding $E$ 's (right graph). This happens because $P$ is the strategy which can fully dispose of $E$ 's. In the model with only $A$ and $E$ strategies the $A$ 's can only significantly shrink long strings of $E$ 's, but once there is an $E$ strategy in the initial state it can never fully disappear, i.e., it can never absorb to a stationary state of only $A$ 's. 

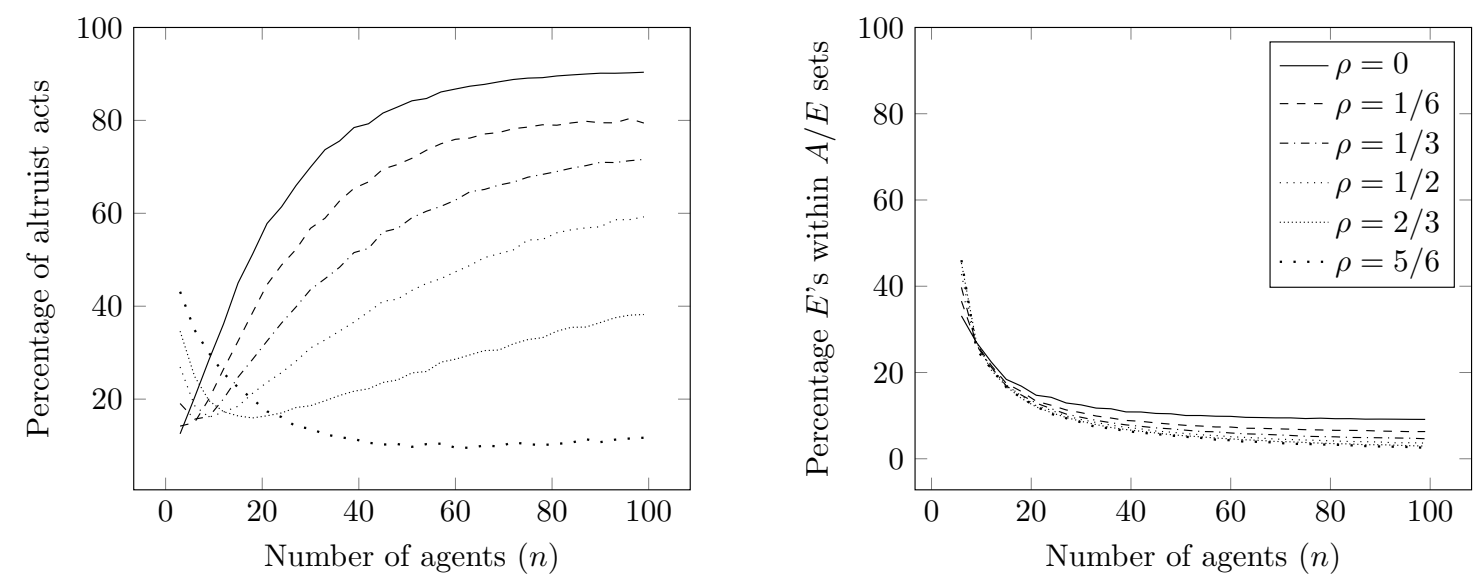

Figure 5: Efficiency as measured by the number of altruist acts (left) and percentage of $E$ strategies within $A / E$ absorbing sets (right).

\subsection{Development of the process}

Using the data gathered over 10,000 simulation runs for a network with $n=99$ agents under default starting conditions for the strategies, the left graph of Figure 6 presents the development of the distribution over strategies used by the agents throughout the process from start to absorption, irrespective of the particular absorbing set that is reached. Note that in order to disentangle short-run, medium-run and long-run effects, the iterations on the $x$-axis follow a square root scale. By default, in the starting state all three strategies have an equal probability of occurrence. The graph ends at the 469-th iteration, when the longest simulation run reached absorption. Information on the absorbing states that were reached earlier is taken into account when deriving the percentages at later iterations. The distribution of absorption times in the right graph explains the overall smoothness after the 200-th iteration, with very few simulations reaching absorption after this number of iterations.

A striking feature of the distribution over strategies presented in the left graph is the sharp decrease in $A$ and $P$ strategies in the first few periods. Strategy $A$ suffers a great loss in numbers from the first to the second iteration, which is consistent with strategy $E$ replacing $A$ and on occasion reaching an all- $E$ absorbing state when the $A$ 's are not clustered. After segregated groups of $A$ 's have been formed they are able to overpower the $E$ 's, after which only small highly resistant pockets of $E$ 's are left within lengthy $A$-strings. At this point the relatively dormant $P$ 's that are left in states which have not reached absorption can take advantage of the $A$ 's. This development is also suggested by the distribution over absorption times (right graph), which exhibits peaks at roughly the average absorption times for the three strategies as suggested by Figure 3, namely at around period 40 for $A / E$ sets, around 50 for $E$ states and around 200 for $P$ states. 

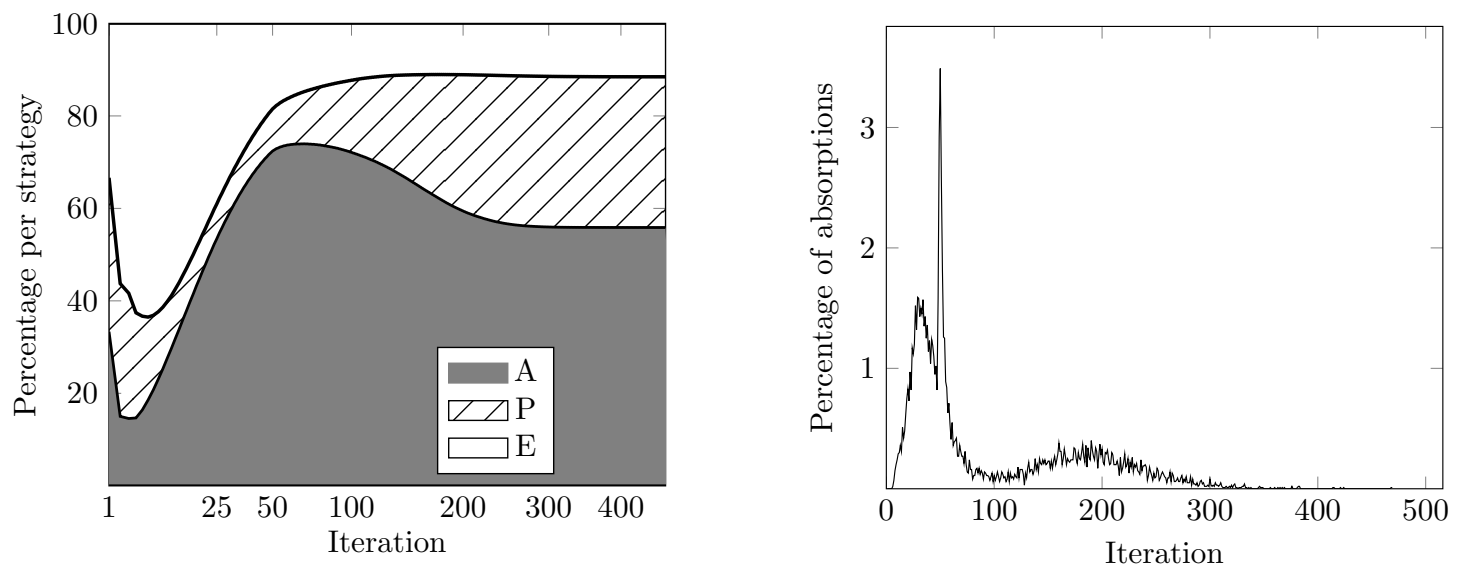

Figure 6: The left graph presents the development of the distribution over strategies used by the agents throughout the process from start to absorption. The right graph presents for each iteration the probability of absorption. Both graphs are based on $n=99$ agents and 10,000 simulation runs.

\subsection{Varying the probability of segregation}

One of the characteristics used to analyze the initial states and how they influence the probability of reaching a particular absorbing set is the index of segregation introduced by Ballester and Vorsatz (2014). This measure, defined for general networks, captures the likelihood that a node of a certain type (in this case, the agent holding a particular strategy) ends his random walk along the links of the network at a node of the same type (in this case, an agent holding the same strategy), where the walk could be terminated at every step with an exogenously given probability. ${ }^{5}$

A gradual increase in the probability of segregation of all three strategies is simulated by increasing the probability (denoted here by $\alpha$ ) that an agent with one strategy would be followed by an agent with the same strategy when constructing the starting state. That is, after the first agent is seeded with one of the strategies, every following agent is assigned the same strategy as the previous agent with probability $\alpha$ and each of the other strategies with probability $\frac{1}{2}(1-\alpha)$. The value of $\alpha$ is varied between $\alpha=0$ (i.e., agents with the same strategy cannot be next to each other, except possibly the first and last agent) to $\alpha=5 / 6$ in steps of $1 / 6$. Figure 7 presents the distributions over strategies in the absorbing state when designing the starting state as just described, for the various values of $\alpha$.

The figure shows that that increasing the probability of segregation increases the chances of the $P$ strategy to take over the circle and overall decreases this probability for the $E$ strategy. This can be explained by clusters of $A$ 's predictably overpowering the $E$ 's after

\footnotetext{
${ }^{5}$ In the simple setting of this model, counting the number of $>1$-strings of different strategies in the starting state gives similar indications to the index of segregation about the probability that a certain absorbing state is reached.
} 


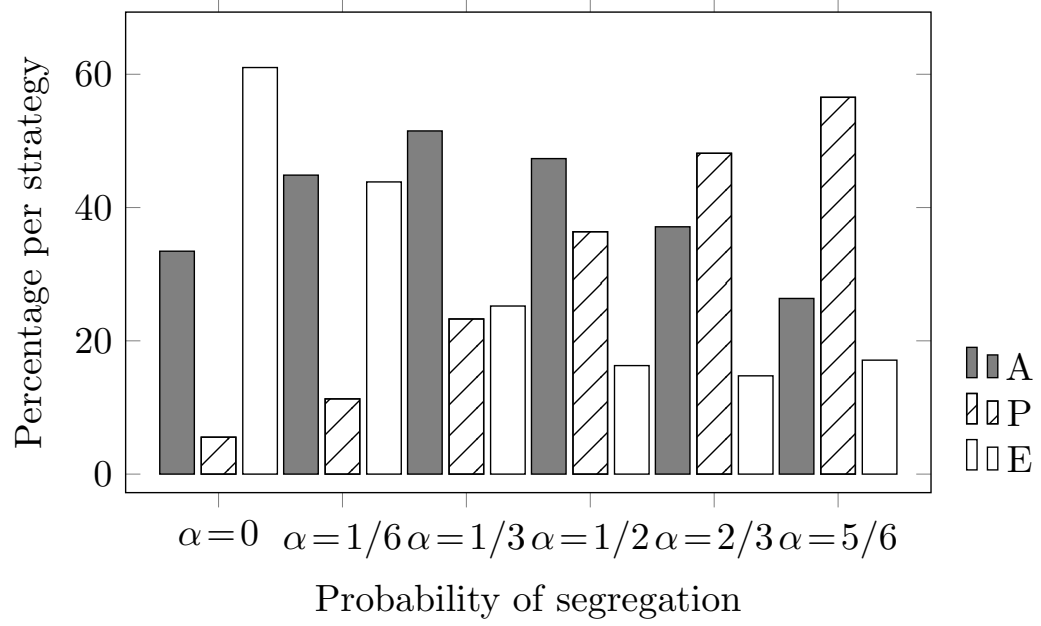

Figure 7: Strategies in the absorbing state varying the segregation probability $(n=60)$.

which they give in to the $P$ 's. For $\alpha=5 / 6$ this effect is offset by the fact that too many $P$ 's are susceptible to free-riding by the $E$ 's. The effect for the $A$ strategy is more complex in the sense that if too scattered, the $A$ 's cannot benefit from cooperating with each other, while if they are too clustered, they could be taken over by the $P$ 's, leaving the circle more vulnerable to the E's.

\section{Conclusion}

This paper presents a local interaction model where agents on a circle use a naïve imitation decision rule, by adopting in each following period the strategy that has provided the highest average payoff from the ones in their observed neighborhood in the current period. The new element in this model is its relative departure from the dichotomous "altruist/egoist" framework that is frequently implemented to study cooperation by introducing a partially altruistic strategy. It allows a middle option between the two extremes, giving the agents the option to cooperate with only one of their neighbors. This feature gives the model a "rock-paper-scissors" flavor, whereby every strategy's propagation is inhibited by one other strategy and propped up by the third one.

The absorbing sets of the Markov process resulting from the imitation dynamic are theoretically characterized and further analyzed by means of computer simulations. The partially cooperative strategy does not coexist with the other two strategies in any absorbing states. The introduction of the partially cooperative strategy has interesting consequences for the development of the process: it hinders the propagation of the altruist strategy and enables the spread of the egoists, and this effect is much stronger than the influence it has on its own diffusion in the population.

On average, the process of reaching absorption could be divided into several phases. 
Firstly, there is a swift elimination of isolated altruists, which solidifies them in bigger blocks. Secondly, they slowly start gaining ground at the expense of big neighboring clusters of egoists. Once this stage is completed, the remaining groups of agents with the partial strategy slowly take over the remaining non-absorbed altruists.

Finally, somewhat contrary to the usual conditions for sustaining altruism, where clusters of altruists in a local interaction model are generally seen as favoring altruism, this model suggests that while it is true that very isolated altruists cannot survive, if they are in too big groups at the beginning of the process, this would benefit the partial strategy and in time even enable the egoists. 


\section{A Proofs}

To ease the notation, $\pi$ denotes $\pi(d)$ throughout the Appendix.

\section{A.1 No splitting of $P$-strings}

Lemma 2. Let $[a, a+k-1]$ be a $k P$-string of the state $x \in X, d \in D(x)$ such that, for every $i \in[a, a+k-1], d_{i}=d_{i}^{*}$ and $y=f(d)$. Then $[a, a+k-1]$ does not split.

Proof. First consider the case $k=1$. Without loss of generality, let $x \in X$ be such that $a=2$, so $x_{2}=P$ and $x_{1}, x_{3} \neq P$. The statement of the lemma is true if one of the following two cases occurs:

(i) $y_{2}=P$.

(ii) $y_{2} \neq P$ and $\left(y_{1} \neq P\right.$ or $\left.y_{3} \neq P\right)$.

Up to symmetries, Cases A.1-A.3 cover all possible segments of 3 agents that contain a $1 P$-string.

Case A.1: $\left(x_{1}, x_{2}, x_{3}\right)=(A, P, A)$

It holds that $d_{2}^{*}=L, \pi_{1} \leq 2-2 c, \pi_{2}=2-c$ and $\pi_{3} \leq 1-2 c$, so $y_{2}=P$.

Case A.2: $\left(x_{1}, x_{2}, x_{3}\right)=(E, P, E)$

It holds that $d_{2}^{*}=L, \pi_{1} \geq 1, \pi_{2}=-c$ and $\pi_{3} \geq 0$, so $y_{1} \neq P$ and $y_{2}=E$.

Case A.3: $\left(x_{1}, x_{2}, x_{3}\right)=(A, P, E)$

It holds that $d_{2}^{*}=L, \pi_{1} \in\{1-2 c, 2-2 c\}, \pi_{2}=1-c$ and $\pi_{3} \in\{0,1\}$. Three cases can be distinguished:

(i) $\pi_{1}=1-2 c$ and $\pi_{3}=0: y_{2}=P$.

(ii) $\pi_{1}=1-2 c$ and $\pi_{3}=1: y_{2}=E$ and $d_{4} \in\{A, L\}$. It follows that $\pi_{4} \leq 1-c$ and $y_{3}=E$.

(iii) $\pi_{1}=2-2 c$ and $\pi_{3} \in\{0,1\}: y_{2}=A$ and $d_{0} \in\{A, R\}$. It follows that $\pi_{0} \geq 1-2 c$ and $y_{1}=A$.

Next, consider the cases $k \neq 1,3$. Without loss of generality, let $x \in X$ be such that $a=2$, so $x_{1} \neq P, x_{2}=\cdots=x_{k+1}=P$ and $x_{k+2} \neq P$. Then, $y_{2}=P$ or $\left(y_{2} \neq P\right.$ and $\left.y_{1} \neq P\right)$. The same argument can be used to show that $y_{k+1}=P$ or $\left(y_{k+1} \neq P\right.$ and $\left.y_{k+2} \neq P\right)$. It holds that $d_{2}^{*}=R, d_{3}^{*}=L, \pi_{2} \geq 1-c$ and $\pi_{3} \geq 1-c$. If $x_{1}=A$, then $\pi_{1} \leq 1-2 c$, so $y_{2}=P$. Assume $x_{1}=E$ and $y_{2} \neq P$. It follows that $\pi_{1}=1$, so $y_{1}=E$.

Finally, consider the case $k=3$. Without loss of generality, let $x \in X$ be such that $a=2$, so $x_{1} \neq P, x_{2}=x_{3}=x_{4}=P$ and $x_{5} \neq P$. It holds that $d_{2}^{*}=R, d_{3}^{*}=R$, and $d_{4}^{*}=L$. Since $d_{3}^{*}=R$ and $d_{4}^{*}=L$, the conclusion that $y_{4}=P$ or $\left(y_{4} \neq P\right.$ and $\left.y_{5} \neq P\right)$ follows as for 
the case $k \neq 1,3$. It suffices to show that $y_{2}=P$ or $\left(y_{2} \neq P\right.$ and $\left.y_{1} \neq P\right)$. If $x_{1}=A$, then $\pi_{1} \leq 1-2 c, \pi_{2}=1-c$ and $\pi_{3}=2-c$, so $y_{2}=P$. Assume $x_{1}=E$ and $y_{2} \neq P$. Since $\pi_{2}=-c$ and $\pi_{3}=2-c, y_{2} \neq P$ implies $\pi_{1}=1$. It then follows that $y_{1} \neq P$.

\section{A.2 1P-strings}

Let $\{a\}$ be a $1 P$-string of the state $x \in X$, so agents $a-1$ and $a+1$ do not use strategy $P$. In case also agents $a-2$ and $a+2$ do not use strategy $P$, we call the segment $[a-2, a+2]$ a $1 P$-segment. Lemma 5 deals with this situation.

Lemma 5. Let $\{a\}$ be a $1 P$-string within a $1 P$-segment of the state $x \in X$. Then one of the following two cases occurs:

(i) there is $y \in F(x)$ such that $p(y) \leq p(x)$ and $y$ contains a $2 P$-string;

(ii) there is $y \in F(x) \cup F^{2}(x)$ such that $p(y) \leq p(x)-1$.

Proof. Table 4 lists all possible $1 P$-segments, omitting symmetric equivalent possibilities. Cases A.4-A.8 below cover all these possible $1 P$-segments and the table makes clear which possibilities are covered by which of these cases. Moreover, the table makes explicit for each possible $1 P$-segment what can happen to the $1 P$-string in this segment. The development of the $1 P$-segments is illustrated in Figure 8.

Case A.4: $\left(x_{1}, x_{2}, x_{3}, x_{4}\right)=(A, A, P, A)$

Take $d_{3}=L$, for $i \neq 3, d_{i}=d_{i}^{*}$, and $y=f(d)$. Then $\pi_{1} \leq 2-2 c, \pi_{2}=2-2 c, \pi_{3}=2-c$ and $\pi_{4} \leq 1-2 c$, so $\left(y_{2}, y_{3}\right)=(P, P)$ and the $1 P$-string $\{3\}$ grows. Lemma 3 implies $p(y) \leq p(x)$.

Case A.5: $\left(x_{1}, x_{2}, x_{3}, x_{4}\right)=(E, E, P, E)$

Take $d_{3}=R$, for $i \neq 3, d_{i}=d_{i}^{*}$, and $y=f(d)$. Then $\pi_{1} \geq 0, \pi_{2}=0, \pi_{3}=-c, \pi_{4} \in\{1,2\}$ and $\pi_{5}<1$ if $x_{5} \neq E$ and $\pi_{5} \in\{0,1\}$ if $x_{5}=E$. In any case $\left(y_{2}, y_{3}, y_{4}\right)=(E, E, E)$ and the $1 P$-string $\{3\}$ disappears. Lemma 3 implies $p(y) \leq p(x)-1$.

Case A.6: $\left(x_{1}, x_{2}, x_{3}\right)=(A, E, P)$

Take $d_{3}=R$, for $i \neq 3, d_{i}=d_{i}^{*}$, and $y=f(d)$. Then $\pi_{1} \leq 1-2 c$ and $\pi_{2}=1$.

(i) $\left(x_{4}, x_{5}\right)=(A, A)$ : then $\pi_{3}=1-c, \pi_{4}=2-2 c$ and $\pi_{5} \geq 1-2 c$, so $\left(y_{2}, y_{3}, y_{4}\right)=(E, A, A)$.

(ii) $\left(x_{4}, x_{5}\right)=(A, E)$ : then $\pi_{3}=1-c, \pi_{4}=1-2 c$ and $\pi_{5} \geq 1$, so $\left(y_{2}, y_{3}, y_{4}\right)=(E, E, E)$.

(iii) $x_{4}=E$ : then $\pi_{3}=-c, \pi_{4} \geq 1$ and $\pi_{5} \leq 1$, so $\left(y_{2}, y_{3}, y_{4}\right)=(E, E, E)$.

In all three subcases above the $1 P$-string $\{3\}$ disappears. Lemma 3 implies $p(y) \leq p(x)-1$.

Case A.7: $\left(x_{1}, x_{2}, x_{3}\right)=(E, A, P)$

Take $d_{3}=L$, for $i \neq 3, d_{i}=d_{i}^{*}$, and $y=f(d)$. Then $\pi_{0} \leq 1, \pi_{1} \geq 1$ and $\pi_{2}=1-2 c$. 
(i) $\left(x_{4}, x_{5}\right)=(A, E)$ : then $\pi_{3}=2-c, \pi_{4}=-2 c, \pi_{5} \geq 1$ and $\pi_{6} \leq 1$, so $y_{1} \neq P, y_{2} \in\{P, E\}$, $y_{3}=P, y_{4} \in\{P, E\}$ and $y_{5}=E$. Lemma 3 implies $p(y) \leq p(x)$. Either the $1 P$-string $\{3\}$ grows or $[1,5]$ is a $1 P$-segment of $y$ that satisfies the conditions of Case A.5, so there is $z \in F(y)$ such that the $1 P$-string $\{3\}$ disappears and $p(z) \leq p(y)-1$.

(ii) $\left(x_{4}, x_{5}\right)=(E, E)$ : then $\pi_{3}=1-c, \pi_{4}=0$ and $\pi_{5} \leq 1$, so $\left(y_{2}, y_{3}, y_{4}\right)=(E, P, P)$ and the $1 P$-string $\{3\}$ grows. Lemma 3 implies $p(y) \leq p(x)$.

Case A.8: $\left(x_{1}, x_{2}, x_{3}, x_{4}, x_{5}\right)=(A, A, P, E, E)$

Take $d_{3}=R$, for $i \neq 3, d_{i}=d_{i}^{*}$, and $y=f(d)$. Then $\pi_{1} \in\{1-2 c, 2-2 c\}, \pi_{2}=1-2 c$, $\pi_{3}=1-c, \pi_{4}=1$ and $\pi_{5} \in\{0,1\}$. It is useful to distinguish a few cases here:

(i) $\pi_{1}=1-2 c$ : then $d_{0} \in\{E, L\}$, so $\pi_{0} \geq 1-c$ and $y_{1} \in\{E, P\},\left(y_{2}, y_{3}\right)=(P, E)$ and $\left(y_{4}, y_{5}\right) \in\{(E, E),(P, P),(P, E)\}$.

If $\left(y_{1}, y_{2}, y_{3}, y_{4}, y_{5}\right)=(P, P, E, P, P)$, then the $1 P$-string $\{3\}$ disappears and it follows by Lemma 3 that $p(y) \leq p(x)-1$.

If $\left(y_{1}, y_{2}, y_{3}, y_{4}, y_{5}\right)=(E, P, E, P, P)$, then it holds by Lemma 3 that $p(y) \leq p(x)$. We can proceed as in Case A.5. Take $d_{2}^{\prime}=L$ and, for $i \neq 2, d_{i}^{\prime}=d_{i}^{*}$. For $z=f\left(d^{\prime}\right)$ it holds that $\left(z_{1}, z_{2}, z_{3}, z_{4}\right)=(E, E, P, P)$, so the $1 P$-string $\{2\}$ disappears and it follows by Lemma 3 that $p(z) \leq p(y)-1$. The case $\left(y_{1}, y_{2}, y_{3}, y_{4}, y_{5}\right)=(P, P, E, P, E)$ follows by a symmetric argument.

If $\left(y_{1}, y_{2}, y_{3}, y_{4}, y_{5}\right)=(E, P, E, P, E)$, then it holds by Lemma 3 that $p(y) \leq p(x)+1$. The construction that follows is also used in Case A.10. Take $d_{2}^{\prime}=L, d_{4}^{\prime}=R$, and, for $i \neq 2,4, d_{i}^{\prime}=d_{i}^{*}$. For $z=f\left(d^{\prime}\right)$ it holds that $\left(z_{1}, z_{2}, z_{3}, z_{4}, z_{5}\right)=(E, E, E, E, E)$, so the $1 P$-strings $\{2\}$ and $\{4\}$ disappear and by Lemma 3 it holds that $p(z) \leq p(y)-2$.

If $\left(y_{1}, y_{2}, y_{3}, y_{4}\right)=(E, P, E, E)$, then it follows by Lemma 3 that $p(y) \leq p(x)$. Since $x_{1}=x_{2} \neq E$, we have $x_{0}=E, \pi_{0} \geq 1$, and $y_{0} \neq P$. Now Case A.5 applies and there is $z \in F(y)$ such that the $1 P$-string $\{2\}$ disappears and $p(z) \leq p(y)-1$.

(ii) $\pi_{1}=2-2 c, \pi_{5}=0$ : then $\left(y_{2}, y_{3}, y_{4}\right)=(A, E, P), y_{5} \in\{P, E\}$. Lemma 3 implies $p(y) \leq$ $p(x)$. Either the $1 P$-string $\{3\}$ grows or $\left(y_{2}, y_{3}, y_{4}, y_{5}\right)=(A, E, P, E)$. We can proceed as in Case A.6. Take $d_{4}^{\prime}=R$, for $i \neq 4, d_{i}=d_{i}^{*}$, and $z=f\left(d^{\prime}\right)$. It holds that $\pi_{2}\left(d^{\prime}\right) \leq 1-2 c$, $\pi_{3}\left(d^{\prime}\right)=1, \pi_{4}\left(d^{\prime}\right)=-c, \pi_{5}\left(d^{\prime}\right) \geq 1$ and $\pi_{6}\left(d^{\prime}\right) \leq 1$, so $\left(z_{3}, z_{4}, z_{5}\right)=(E, E, E)$ and the $1 P$-string $\{4\}$ disappears. Lemma 3 implies $p(z) \leq p(y)-1$.

(iii) $\pi_{1}=2-2 c, \pi_{5}=1$ : then $\left(y_{2}, y_{3}, y_{4}\right)=(A, E, E)$. The $1 P$-string $\{3\}$ disappears and Lemma 3 implies $p(y) \leq p(x)-1$.

Let $\{a\}$ and $\{b\}$ be $1 P$-strings of the state $x \in X$, where $b=a+2$. We call the segment $[a-1, b+1]$ a $1 P+1 P$-segment. Notice that $x_{a+1}=x_{b-1} \neq P$. Lemma 6 deals with this 


\begin{tabular}{lccl}
\hline Case & Segment $\left(x_{1}, x_{2}, x_{3}, x_{4}, x_{5}\right)$ & Decision $d_{3}$ & Results \\
\hline A.4 & $(A, A, P, A, A)$ & $L$ & $\geq 2 P$ \\
A.4 & $(A, A, P, A, E)$ & $L$ & $\geq 2 P$ \\
A.6 & $(A, A, P, E, A)$ & $L$ & $0 P$ \\
A.8 & $(A, A, P, E, E)$ & $R$ & $0 P ; \geq 2 P$ \\
A.6 & $(A, E, P, A, E)$ & $R$ & $0 P$ \\
A.6 & $(A, E, P, E, A)$ & $R$ & $0 P$ \\
A.5 & $(A, E, P, E, E)$ & $L$ & $0 P$ \\
A.7 & $(E, A, P, A, E)$ & $L$ & $0 P ; \geq 2 P$ \\
A.7 & $(E, A, P, E, E)$ & $L$ & $\geq 2 P$ \\
A.5 & $(E, E, P, E, E)$ & $R$ & $0 P$ \\
\hline
\end{tabular}

Table 4: All possible $1 P$-segments, omitting symmetric equivalent possibilities, and what can happen with the $1 P$-string within the segment.

situation. The case where a $1 P$-string does not border another $1 P$-string, but does border a $\geq 2 P$-string from at least one side (i.e. there is exactly one agent between the $1 P$-string and a neighboring $\geq 2 P$-string, is addressed in Appendices A.3-A.5, where $\geq 2 P$-strings are studied.

Lemma 6. Let $\{a\}$ and $\{b\}$ be $1 P$-strings within a $1 P+1 P$-segment of the state $x \in X$. Then one of the following two cases occurs:

(i) there is $y \in F(x) \cup F^{2}(x)$ such that $p(y) \leq p(x)$ and $y$ contains $a \geq 2 P$-string;

(ii) there is $y \in F(x) \cup F^{2}(x) \cup F^{3}(x)$ such that $p(y) \leq p(x)-1$.

Proof. Table 5 lists all possible $1 P+1 P$-segments, omitting symmetric equivalent possibilities. Cases A.9-A.13 below cover all these possible $1 P+1 P$-segments and the table makes clear which possibilities are covered by which of these cases. Moreover, the table makes explicit for each possible $1 P+1 P$-segment what can happen to the $1 P$-strings in this segment. The development of the $1 P+1 P$-segments is illustrated in Figure 8 .

Case A.9: $\left(x_{1}, x_{2}, x_{3}, x_{4}\right)=(A, P, A, P)$

Take $d_{2}=L, d_{4}=R$, for $i \neq 2,4, d_{i}=d_{i}^{*}$, and $y=f(d)$. Then $\pi_{1} \geq 1-2 c, \pi_{2}=2-c$, $\pi_{3}=-2 c$ and $\pi_{4} \geq 1-c$, so $\left(y_{2}, y_{3}\right)=(P, P)$ and the $1 P$-string $\{2\}$ grows. Lemma 3 implies $p(y) \leq p(x)$.

Case A.10: $\left(x_{1}, x_{2}, x_{3}, x_{4}, x_{5}\right)=(E, P, E, P, E)$

Take $d_{2}=L, d_{4}=R$, for $i \neq 2,4, d_{i}=d_{i}^{*}$, and $y=f(d)$. Then $\pi_{1}, \pi_{5} \geq 1, \pi_{2}=\pi_{4}=-c$ and $\pi_{3}=0$, so $\left(y_{1}, y_{2}, y_{3}, y_{4}, y_{5}\right)=(E, E, E, E, E)$ and the $1 P$-strings $\{2\}$ and $\{4\}$ disappear. Lemma 3 implies $p(y) \leq p(x)-2$.

Case A.11: $\left(x_{1}, x_{2}, x_{3}, x_{4}, x_{5}\right)=(A, P, E, P, E)$

Take $d_{2}=L, d_{4}=R$, for $i \neq 2,4, d_{i}=d_{i}^{*}$, and $y=f(d)$. Then $\pi_{2}=1-c, \pi_{3}=0, \pi_{4}=-c$ and $\pi_{5} \geq 1$. 
(i) $\pi_{1}=1-2 c$ : then $\left(y_{2}, y_{3}, y_{4}, y_{5}\right)=(P, P, E, E)$. The $1 P$-string $\{2\}$ grows and Lemma 3 implies $p(y) \leq p(x)-1$.

(ii) $\pi_{1}=2-2 c$ : then $\left(y_{1}, y_{2}, y_{3}, y_{4}, y_{5}\right)=(A, A, P, E, E)$. Lemma 3 implies $p(y) \leq p(x)-1 .^{6}$

Case A.12: $\left(x_{1}, x_{2}, x_{3}, x_{4}, x_{5}\right)=(A, P, E, P, A)$

Take $d_{2}=L, d_{4}=R$, for $i \neq 2,4, d_{i}=d_{i}^{*}$, and $y=f(d)$. Then $\pi_{1} \in\{1-2 c, 2-2 c\}, \pi_{2}=1-c$, $\pi_{3}=0, \pi_{4}=1-c$ and $\pi_{5} \in\{1-2 c, 2-2 c\}$. We consider the following cases concerning $\pi_{5}$ (bearing in mind that the cases for $\pi_{1}$ are symmetric):

(i) $\pi_{5}=1-2 c$ : then $d_{6} \in\{R, E\},\left(y_{3}, y_{4}\right)=(P, P), y_{5} \in\{P, E\}$.

(ii) $\pi_{5}=2-2 c$ : then $d_{6} \in\{A, L\},\left(y_{3}, y_{4}, y_{5}\right)=(P, A, A)$.

Keeping the symmetric structure in mind, the segment results in a $\geq 2 P$-string and, by Lemma $3, p(y) \leq p(x)-1$, or a $1 P$-segment and, by Lemma $3, p(y) \leq p(x)-1 .^{7}$

Case A.13: $\left(x_{1}, x_{2}, x_{3}, x_{4}, x_{5}\right)=(E, P, A, P, E)$

Take $d_{2}=L, d_{4}=L$, for $i \neq 2,4, d_{i}=d_{i}^{*}$, and $y=f(d)$. Then $\pi_{1} \in\{1,2\}, \pi_{2}=1-c$, $\pi_{3}=1-2 c, \pi_{4}=1-c$ and $\pi_{5} \in\{0,1\}$. It holds that $y_{1} \in\{P, E\}$ and $\left(y_{2}, y_{3}\right)=(E, P)$.

If $\pi_{5}=0$, then $d_{6} \in\{E, R\}$ and $\pi_{6} \leq 1$, so $\left(y_{4}, y_{5}\right)=(P, P)$. The $1 P$-string $\{4\}$ grows and Lemma 3 implies $p(y) \leq p(x)$.

If $\pi_{5}=1$, then $d_{6} \in\{A, L\}$ and $\pi_{6} \leq 1-c$, so $\left(y_{4}, y_{5}\right)=(E, E)$. Lemma 3 implies $p(y) \leq p(x)$. We can proceed as in Case A.5. Take $d_{2}^{\prime}=L$ and, for $i \neq 2, d_{i}^{\prime}=d_{i}^{*}$. For $z=f\left(d^{\prime}\right)$ it holds that $\left(z_{2}, z_{3}, z_{4}\right)=(E, E, E)$, so the $1 P$-string $\{3\}$ disappears and Lemma 3 implies $p(z) \leq p(y)-1$.

\begin{tabular}{lccl}
\hline Case & Segment $\left(x_{1}, x_{2}, x_{3}, x_{4}, x_{5}\right)$ & Decisions $d_{2}, d_{4}$ & Results \\
\hline A.9 & $(A, P, A, P, A)$ & $L, R$ & $\geq 2 P$ \\
A.9 & $(A, P, A, P, E)$ & $L, R$ & $\geq 2 P$ \\
A.12 & $(A, P, E, P, A)$ & $L, R$ & $\geq 2 P$ \\
A.11 & $(A, P, E, P, E)$ & $L, R$ & $0 P ; \geq 2 P$ \\
A.13 & $(E, P, A, P, E)$ & $L, L$ & $0 P ; \geq 2 P$ \\
A.10 & $(E, P, E, P, E)$ & $L, R$ & $0 P$ \\
\hline
\end{tabular}

Table 5: All possible $1 P+1 P$-segments, omitting symmetric equivalent possibilities.

\section{A.3 $2 P$-strings}

Lemma 7. Let $[a, a+1]$ be a $2 P$-string of the state $x \in X$. Then one of the following two cases occurs:

\footnotetext{
${ }^{6}$ From $y$ we can continue as in Case A.8 and find a state such that the $1 P$-string $\{3\}$ disappears or grows.

${ }^{7}$ Now Case A. 4 applies, leading to a $\geq 2 P$-string in the next iteration.
} 


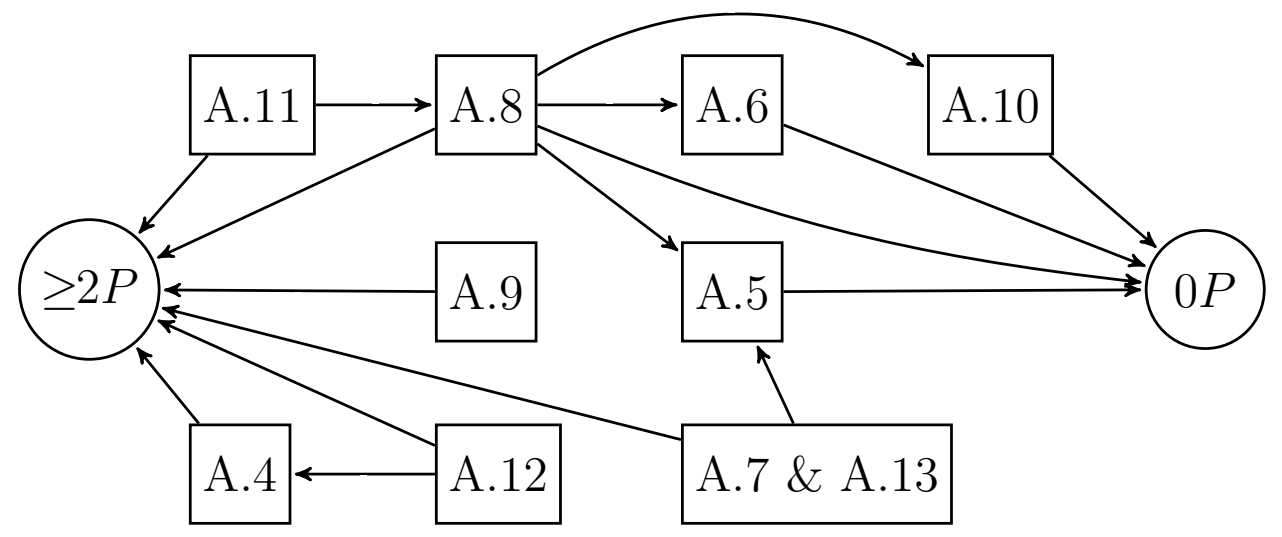

Figure 8: Transforming $1 P$-segments and $1 P+1 P$-segments based on the cases specified in the squares results in the $P$-strings in the circles.

(i) there is $y \in F(x) \cup F^{2}(x)$ such that $p(y) \leq p(x)$ and $y$ contains $a \geq 3 p$-string;

(ii) there is $y \in F(x)$ such that $p(y) \leq p(x)-1$.

Proof. Cases A.14-A.23 below cover all these possible $2 P$-strings. Table 6 lists all possible cases and makes explicit for each case what can happen to the $2 P$-strings. The development of the $2 P$-strings is illustrated in Figure 9.

Case A.14: $d_{1}^{*} \in\{A, R, L\},\left(x_{2}, x_{3}, x_{4}, x_{5}\right)=(A, P, P, A)$ and $d_{6}^{*} \in\{A, R, L\}$

Take $\left(d_{3}, d_{4}\right)=(R, L)$, for $i \neq 3,4, d_{i}=d_{i}^{*}$, and $y=f(d)$. Then $\pi_{1} \geq 1-2 c, \pi_{2} \leq 1-2 c$, $\pi_{3}=2-c, \pi_{4}=2-c, \pi_{5} \leq 1-2 c$ and $\pi_{6} \geq 1-2 c$ and hence $\left(y_{2}, y_{3}, y_{4}, y_{5}\right)=(P, P, P, P)$. The $2 P$-string $[3,4]$ grows and Lemma 3 implies $p(y) \leq p(x)$.

Case A.15: $d_{1}^{*} \in\{A, R, L\},\left(x_{2}, x_{3}, x_{4}, x_{5}\right)=(A, P, P, A)$ and $d_{6}^{*}=E$

Take $\left(d_{3}, d_{4}\right)=(R, L)$, for $i \neq 3,4, d_{i}=d_{i}^{*}$, and $y=f(d)$. Then $\pi_{1} \geq 1-2 c, \pi_{2} \leq 1-2 c$, $\pi_{3}=2-c, \pi_{4}=2-c, \pi_{5}=-2 c$ and $\pi_{6} \geq 1$ and hence $\left(y_{2}, y_{3}, y_{4}\right)=(P, P, P),\left(y_{5}, y_{6}\right) \in$ $\{(P, E),(E, E)\}$. The $2 P$-string $[3,4]$ grows and Lemma 3 implies $p(y) \leq p(x)$.

Case A.16: $d_{1}^{*} \in\{E, L\},\left(x_{2}, x_{3}, x_{4}, x_{5}\right)=(E, P, P, E)$ and $d_{6}^{*} \in\{E, R\}$

Take $\left(d_{3}, d_{4}\right)=(R, L)$, for $i \neq 3,4, d_{i}=d_{i}^{*}$, and $y=f(d)$. Then $\pi_{1} \leq 1, \pi_{2}=0, \pi_{3}=1-c$, $\pi_{4}=1-c, \pi_{5}=0$ and $\pi_{6} \leq 1$ and hence $\left(y_{2}, y_{3}, y_{4}, y_{5}\right)=(P, P, P, P)$. The $2 P$-string $[3,4]$ grows and Lemma 3 implies $p(y) \leq p(x)$.

Case A.17: $d_{1}^{*}=E,\left(x_{2}, x_{3}, x_{4}, x_{5}\right)=(A, P, P, A)$ and $d_{6}^{*}=E$

Take $\left(d_{3}, d_{4}\right)=(R, L)$, for $i \neq 3,4, d_{i}=d_{i}^{*}$, and $y=f(d)$. Then $\pi_{1} \geq 1, \pi_{2}=-2 c, \pi_{3}=2-c$, $\pi_{4}=2-c, \pi_{5}=-2 c$ and $\pi_{6} \geq 1$ and hence $\left(y_{1}, y_{2}\right) \in\{(E, P),(E, E)\},\left(y_{3}, y_{4}\right)=(P, P)$ and $\left(y_{5}, y_{6}\right) \in\{(P, E),(E, E)\}$. Lemma 3 implies $p(y) \leq p(x)$. If $\left(y_{1}, y_{2}, y_{3}, y_{4}, y_{5}, y_{6}\right) \neq$ $(E, E, P, P, E, E)$, then the $2 P$-string $[3,4]$ grows. Otherwise, we can apply Case A.16 and find $z \in F(y)$ such that $p(z) \leq p(y)$ and the $2 P$-string [3,4] grows. 
Case A.18: $d_{1}^{*} \in\{A, R, L\},\left(x_{2}, x_{3}, x_{4}, x_{5}\right)=(A, P, P, E)$ and $d_{6}^{*} \in\{E, R\}$

Take $\left(d_{3}, d_{4}\right)=(R, L)$, for $i \neq 3,4, d_{i}=d_{i}^{*}$, and $y=f(d)$. Then $\pi_{1} \geq 1-2 c, \pi_{2} \leq 1-2 c$, $\pi_{3}=2-c, \pi_{4}=1-c, \pi_{5}=0$ and $\pi_{6} \leq 1$ and hence $\left(y_{2}, y_{3}, y_{4}, y_{5}\right)=(P, P, P, P)$. The $2 P$-string $[3,4]$ grows and Lemma 3 implies $p(y) \leq p(x)$.

Case A.19: $d_{1}^{*}=E,\left(x_{2}, x_{3}, x_{4}, x_{5}\right)=(A, P, P, E)$ and $d_{6}^{*} \in\{E, R\}$

Take $\left(d_{3}, d_{4}\right)=(R, L)$, for $i \neq 3,4, d_{i}=d_{i}^{*}$, and $y=f(d)$. Then $\pi_{1} \geq 1, \pi_{2}=-2 c, \pi_{3}=2-c$, $\pi_{4}=1-c, \pi_{5}=0$ and $\pi_{6} \leq 1$ and hence $\left(y_{1}, y_{2}\right) \in\{(E, P),(E, E)\}$ and $\left(y_{3}, y_{4}, y_{5}\right)=(P, P, P)$. The $2 P$-string $[3,4]$ grows and Lemma 3 implies $p(y) \leq p(x)$.

Case A.20: $d_{1}^{*} \in\{A, R, L\},\left(x_{2}, x_{3}, x_{4}, x_{5}\right)=(A, P, P, E)$ and $d_{6}^{*} \in\{A, L\}$

Take $\left(d_{3}, d_{4}\right)=(R, L)$, for $i \neq 3,4, d_{i}=d_{i}^{*}$, and $y=f(d)$. Then $\pi_{1} \geq 1-2 c, \pi_{2} \leq 1-2 c$, $\pi_{3}=2-c, \pi_{4}=1-c, \pi_{5}=1$ and $\pi_{6} \leq 1-c$ and hence $\left(y_{2}, y_{3}, y_{4}, y_{5}\right)=(P, P, P, E)$. The $2 P$-string $[3,4]$ grows and Lemma 3 implies $p(y) \leq p(x)$.

Case A.21: $d_{1}^{*}=E,\left(x_{2}, x_{3}, x_{4}, x_{5}\right)=(A, P, P, E)$ and $d_{6}^{*} \in\{A, L\}$

Take $\left(d_{3}, d_{4}\right)=(L, R)$, for $i \neq 3,4, d_{i}=d_{i}^{*}$, and $y=f(d)$. Then $\pi_{1} \geq 1, \pi_{2}=1-2 c$, $\pi_{3}=1-c, \pi_{4}=-c, \pi_{5}=2$ and $\pi_{6} \leq 1-2 c$ and $\left(y_{2}, y_{3}, y_{4}, y_{5}\right)=(E, A, E, E)$. The $2 P$-string $[3,4]$ disappears and Lemma 3 implies $p(y) \leq p(x)-1$.

Case A.22: $d_{1}^{*} \in\{E, L\},\left(x_{2}, x_{3}, x_{4}, x_{5}\right)=(E, P, P, E)$ and $d_{6}^{*} \in\{A, L\}$

Take $\left(d_{3}, d_{4}\right)=(L, R)$, for $i \neq 3,4, d_{i}=d_{i}^{*}$, and $y=f(d)$. Then $\pi_{1} \leq 1, \pi_{2}=1, \pi_{3}=-c$, $\pi_{4}=-c, \pi_{5}=2$ and $\pi_{6} \leq 1-c$ and $\left(y_{2}, y_{3}, y_{4}, y_{5}\right)=(E, E, E, E)$. The $2 P$-string [3,4] disappears and Lemma 3 implies $p(y) \leq p(x)-1$.

Case A.23: $d_{1}^{*} \in\{A, R\},\left(x_{2}, x_{3}, x_{4}, x_{5}\right)=(E, P, P, E)$ and $d_{6}^{*} \in\{A, L\}$

Take $\left(d_{3}, d_{4}\right)=(L, R)$, for $i \neq 3,4, d_{i}=d_{i}^{*}$, and $y=f(d)$. Then $\pi_{1} \leq 1-c, \pi_{2}=2, \pi_{3}=-c$, $\pi_{4}=-c, \pi_{5}=2, \pi_{6} \leq 1-c$ and $\left(y_{2}, y_{3}, y_{4}, y_{5}\right)=(E, E, E, E)$. The $2 P$-string $[3,4]$ disappears and Lemma 3 implies $p(y) \leq p(x)-1$.

\begin{tabular}{|c|c|c|c|c|c|c|c|c|}
\hline \multirow{2}{*}{\multicolumn{2}{|c|}{$\left(x_{3}, x_{4}\right)=(P, P)$}} & \multicolumn{7}{|c|}{$\left(d_{5}^{*}, d_{6}^{*}\right)=$} \\
\hline & & $(A, A / R / L)$ & \multicolumn{2}{|c|}{$(A, E)$} & \multicolumn{2}{|c|}{$(E, E / R)$} & \multicolumn{2}{|c|}{$(E, A / L)$} \\
\hline$\|$ & $(A / R / L, A)$ & $\mathrm{g} \quad(\mathrm{A} .14)$ & $\mathrm{g}$ & (A.15) & $\mathrm{g}$ & (A.18) & $\mathrm{g}$ & (A.20) \\
\hline$\overparen{* N}$ & $(E, A)$ & & $\mathrm{g}$ & (A.17) & $\mathrm{g}$ & (A.19) & d & (A.21) \\
\hline 急 & $(E / L, E)$ & & & & $\mathrm{g}$ & (A.16) & & (A.22) \\
\hline$\sqrt{2}$ & $(A / R, E)$ & & & & & & d & (A.23) \\
\hline
\end{tabular}

Table 6: Strings resulting from $2 P$-strings surrounded from the left by the decisions in the columns and from the right by the decisions in the rows. Abbreviations ' $\mathrm{g}$ ' and 'd' in the cells are used to identify whether the $P$-string will be growing or disappearing. Relevant cases are in parentheses. 


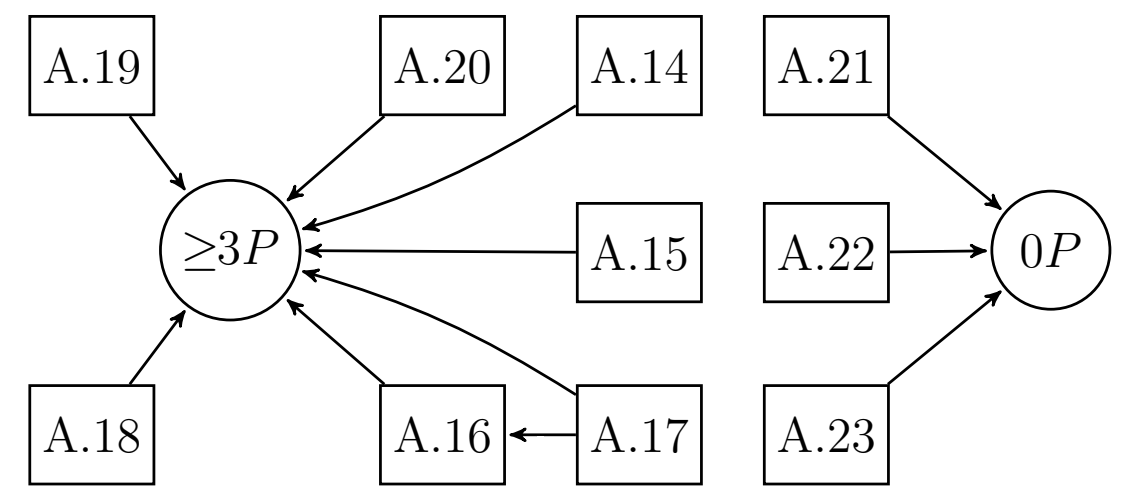

Figure 9: Transforming $2 P$-strings based on the cases specified in the squares results in the $P$-strings in the circles.

\section{A.4 $3 P$-strings}

Lemma 8. Let $n \geq 4$ and let $[a, a+2]$ be a $3 P$-string of the state $x \in X$. Then one of the following two cases occurs:

(i) there is $y \in F(x) \cup F^{2}(x)$ such that $p(y) \leq p(x)$ and $y$ contains $a \geq 4 P$-string;

(ii) there is $y \in F^{2}(x) \cup F^{3}(x)$ such that $p(y) \leq p(x)-1$.

Proof. Cases A.24-A.33 describe all possible 3P-strings. Table 7 lists all possible cases and makes explicit for each possibility what can happen to the $3 P$-strings. The development of the $3 P$-strings is illustrated in Figure 10.

Case A.24: $d_{1}^{*} \in\{A, R, L\},\left(x_{2}, x_{3}, x_{4}, x_{5}, x_{6}\right)=(A, P, P, P, A)$ and $d_{7}^{*} \in\{A, R, L\}$

Take $\left(d_{3}, d_{4}, d_{5}\right)=(R, R, L)$, for $i \neq 3,4,5, d_{i}=d_{i}^{*}$, and $y=f(d)$. Then $\pi_{1} \geq 1-2 c$, $\pi_{2} \leq 1-2 c, \pi_{3}=1-c, \pi_{4}=2-c, \pi_{5}=2-c, \pi_{6} \leq 1-2 c$ and $\pi_{7} \geq 1-2 c$ and hence $\left(y_{3}, y_{4}, y_{5}, y_{6}\right)=(P, P, P, P)$. The $3 P$-string $[3,5]$ grows and Lemma 3 implies $p(y) \leq p(x)$.

Case A.25: $d_{1}^{*} \in\{A, R, L\},\left(x_{2}, x_{3}, x_{4}, x_{5}, x_{6}\right)=(A, P, P, P, A)$ and $d_{7}^{*}=E$

Take $\left(d_{3}, d_{4}, d_{5}\right)=(R, L, L)$, for $i \neq 3,4,5, d_{i}=d_{i}^{*}$, and $y=f(d)$. Then $\pi_{1} \geq 1-2 c$, $\pi_{2} \leq 1-2 c, \pi_{3}=2-c, \pi_{4}=2-c, \pi_{5}=1-c, \pi_{6}=-2 c$ and $\pi_{7} \geq 1$ and hence $\left(y_{2}, y_{3}, y_{4}, y_{5}\right)=(P, P, P, P)$. The $3 P$-string $[3,5]$ grows and Lemma 3 implies $p(y) \leq p(x)$.

Case A.26: $d_{1}^{*} \in\{E, L\},\left(x_{2}, x_{3}, x_{4}, x_{5}, x_{6}\right)=(E, P, P, P, E)$ and $d_{7}^{*} \in\{E, R\}$

Take $\left(d_{3}, d_{4}, d_{5}\right)=(R, R, L)$, for $i \neq 3,4,5, d_{i}=d_{i}^{*}$, and $y=f(d)$. Then $\pi_{1} \leq 1, \pi_{2}=0$, $\pi_{3}=-c, \pi_{4}=2-c, \pi_{5}=1-c, \pi_{6}=0$ and $\pi_{7} \leq 1$ and hence $\left(y_{3}, y_{4}, y_{5}, y_{6}\right)=(P, P, P, P)$. The $3 P$-string $[3,5]$ grows and Lemma 3 implies $p(y) \leq p(x)$.

Case A.27: $d_{1}^{*}=E,\left(x_{2}, x_{3}, x_{4}, x_{5}, x_{6}\right)=(A, P, P, P, A)$ and $d_{7}^{*}=E$

Take $\left(d_{3}, d_{4}, d_{5}\right)=(R, R, L)$, for $i \neq 3,4,5, d_{i}=d_{i}^{*}$, and $y=f(d)$. Then $\pi_{1} \geq 1, \pi_{2}=-2 c$, $\pi_{3}=1-c, \pi_{4}=2-c, \pi_{5}=2-c, \pi_{6}=-2 c$ and $\pi_{7} \geq 1$ and hence $\left(y_{1}, y_{2}, y_{3}, y_{4}, y_{5}\right)=$ 
$(E, E, P, P, P), y_{6} \in\{P, E\}$ and $y_{7}=E$. Lemma 3 implies $p(y) \leq p(x)$. If $\left(y_{1}, y_{2}, y_{3}, y_{4}, y_{5}, y_{6}, y_{7}\right)=$ $(E, E, P, P, P, P, E)$, then the $3 P$-string $[3,5]$ grows. Otherwise, $\left(y_{1}, y_{2}, y_{3}, y_{4}, y_{5}, y_{6}, y_{7}\right)=$ $(E, E, P, P, P, E, E)$ and Case A.26 implies that we can find $z \in F(y)$ such that $p(z) \leq p(y)$ and the $3 P$-string $[3,5]$ grows.

Case A.28: $d_{1}^{*} \in\{A, R, L\},\left(x_{2}, x_{3}, x_{4}, x_{5}, x_{6}\right)=(A, P, P, P, E)$ and $d_{7}^{*} \in\{E, R\}$ Take $\left(d_{3}, d_{4}, d_{5}\right)=(R, L, L)$, for $i \neq 3,4,5, d_{i}=d_{i}^{*}$, and $y=f(d)$. Then $\pi_{1} \geq 1-2 c$, $\pi_{2} \leq 1-2 c, \pi_{3}=2-c, \pi_{4}=2-c, \pi_{5}=-c, \pi_{6}=0$ and $\pi_{7} \leq 1$ and hence $\left(y_{2}, y_{3}, y_{4}, y_{5}\right)=$ $(P, P, P, P)$. The $3 P$-string $[3,5]$ grows and Lemma 3 implies $p(y) \leq p(x)$.

Case A.29: $d_{1}^{*}=E,\left(x_{2}, x_{3}, x_{4}, x_{5}, x_{6}\right)=(A, P, P, P, E)$ and $d_{7}^{*} \in\{E, R\}$

Take $\left(d_{3}, d_{4}, d_{5}\right)=(R, R, L)$, for $i \neq 3,4,5, d_{i}=d_{i}^{*}$, and $y=f(d)$. Then $\pi_{1} \geq 1, \pi_{2}=-2 c$, $\pi_{3}=1-c, \pi_{4}=2-c, \pi_{5}=1-c, \pi_{6}=0$ and $\pi_{7} \leq 1$ and hence $\left(y_{1}, y_{2}, y_{3}, y_{4}, y_{5}, y_{6}\right)=$ $(E, E, P, P, P, P)$. The $3 P$-string $[3,5]$ grows and Lemma 3 implies $p(y) \leq p(x)$.

Case A.30: $d_{1}^{*} \in\{E, L\},\left(x_{2}, x_{3}, x_{4}, x_{5}, x_{6}\right)=(E, P, P, P, E)$ and $d_{7}^{*} \in\{A, L\}$

Take $\left(d_{3}, d_{4}, d_{5}\right)=(L, R, R)$, for $i \neq 3,4,5, d_{i}=d_{i}^{*}$, and $y=f(d)$. Then $\pi_{1} \leq 1, \pi_{2}=1$, $\pi_{3}=-c, \pi_{4}=-c, \pi_{5}=1-c, \pi_{6}=2$ and $\pi_{7} \leq 1-c$ and $\left(y_{2}, y_{3}, y_{4}, y_{5}, y_{6}\right)=(E, E, P, E, E)$. Lemma 3 implies $p(y) \leq p(x)$. By Case A.5 we can find $z \in F(y)$ such that $p(z) \leq p(y)-1$ and the $1 P$-string $\{4\}$ disappears.

Case A.31: $d_{1}^{*} \in\{A, R, L\},\left(x_{2}, x_{3}, x_{4}, x_{5}, x_{6}\right)=(A, P, P, P, E)$ and $d_{7}^{*} \in\{A, L\}$

Take $\left(d_{3}, d_{4}, d_{5}\right)=(R, L, L)$, for $i \neq 3,4,5, d_{i}=d_{i}^{*}$, and $y=f(d)$. Then $\pi_{1} \geq 1-2 c$, $\pi_{2} \leq 1-2 c, \pi_{3}=2-c, \pi_{4}=2-c, \pi_{5}=-c, \pi_{6}=1$ and $\pi_{7} \leq 1-c$ and hence $\left(y_{2}, y_{3}, y_{4}, y_{5}, y_{6}\right)=$ $(P, P, P, E, E)$.

If $y_{1}=P$, then Lemma 3 implies $p(y) \leq p(x)-1$ and $y$ contains a $\geq 4 P$-string.

If $y_{1}=A$, then Lemma 3 implies $p(y) \leq p(x)$. Moreover, either Case A.28 or Case A.29 applies, so there is $z \in F(y)$ such that the $3 P$-string $[2,4]$ grows and $p(z) \leq p(y)$.

If $y_{1}=E$, then Lemma 3 implies $p(y) \leq p(x)$. Either Case A.26 applies and there is $z \in F(y)$ such that $p(z) \leq p(y)$ and the $3 P$-string $[2,4]$ grows or Case A.30 applies and there is $z \in F(y)$ such that $p(z) \leq p(y)$ and $\left(z_{1}, z_{2}, z_{3}, z_{4}, z_{5}\right)=(E, E, P, E, E)$ and there is $w \in F(z)$ such that $p(w) \leq p(z)-1$ and the $1 P$-string $\{3\}$ disappears.

Case A.32: $d_{1}^{*}=E,\left(x_{2}, x_{3}, x_{4}, x_{5}, x_{6}\right)=(A, P, P, P, E)$ and $d_{7}^{*} \in\{A, L\}$

Take $\left(d_{3}, d_{4}, d_{5}\right)=(R, R, L)$, for $i \neq 3,4,5, d_{i}=d_{i}^{*}$, and $y=f(d)$. Then $\pi_{1} \geq 1, \pi_{2}=-2 c$, $\pi_{3}=1-c, \pi_{4}=2-c, \pi_{5}=1-c, \pi_{6}=1$ and $\pi_{7} \leq 1$ and hence $\left(y_{1}, y_{2}, y_{3}, y_{4}, y_{5}, y_{6}\right)=$ $(E, E, P, P, P, E)$. Lemma 3 implies $p(y) \leq p(x)$. Either Case A.26 applies and there is $z \in F(y)$ such that the $3 P$-string $[3,5]$ grows and $p(z) \leq p(y)$ or Case A.30 applies and there is $z \in F(y)$ such that $p(z) \leq p(y)$ and $\left(z_{2}, z_{3}, z_{4}, z_{5}, z_{6}\right)=(E, E, P, E, E)$ and there is $w \in F(z)$ such that $p(w) \leq p(z)-1$ and the $1 P$-string $\{4\}$ disappears.

Case A.33: $d_{1}^{*} \in\{A, R\},\left(x_{2}, x_{3}, x_{4}, x_{5}, x_{6}\right)=(E, P, P, P, E)$ and $d_{7}^{*} \in\{A, L\}$ 
Take $\left(d_{3}, d_{4}, d_{5}\right)=(L, R, R)$, for $i \neq 3,4,5, d_{i}=d_{i}^{*}$, and $y=f(d)$. Then $\pi_{1} \leq 1-c, \pi_{2}=2$, $\pi_{3}=-c, \pi_{4}=-c, \pi_{5}=1-c, \pi_{6}=2$ and $\pi_{7} \leq 1-c$ and $\left(y_{2}, y_{3}, y_{4}, y_{5}, y_{6}\right)=(E, E, P, E, E)$. Lemma 3 implies $p(y) \leq p(x)$. By Case A.5 we can find $z \in F(y)$ such that $p(z) \leq p(y)-1$ and the $1 P$-string $\{4\}$ disappears.

\begin{tabular}{|c|c|c|c|c|c|}
\hline \multirow{2}{*}{\multicolumn{2}{|c|}{$\left(x_{3}, x_{4}, x_{5}\right)=(P, P, P)$}} & \multicolumn{4}{|c|}{$\left(d_{6}^{*}, d_{7}^{*}\right)=$} \\
\hline & & $(A, A / R / L)$ & $(A, E)$ & $(E, E / R)$ & $(E, A / L)$ \\
\hline$\|$ & $(A / R / L, A)$ & $\mathrm{g} \quad(\mathrm{A} .24)$ & $\mathrm{g} \quad(\mathrm{A} .25)$ & $\mathrm{g} \quad(\mathrm{A} .28)$ & $\mathrm{g} ; \mathrm{d}(\mathrm{A} .31)$ \\
\hline$\overbrace{x \rightarrow 1}^{11}$ & $(E, A)$ & & (A.27) & (A.29) & $\mathrm{g} ; \mathrm{d}(\mathrm{A} .32)$ \\
\hline $\begin{array}{l}0 \\
*=1\end{array}$ & $(E / L, E)$ & & & (A.26) & d (A.30) \\
\hline$\Xi$ & $(A / R, E)$ & & & & (A.33) \\
\hline
\end{tabular}

Table 7: Strings resulting from $3 P$-strings surrounded from the left by the decisions in the columns and from the right by the decisions in the rows. Abbreviations ' $\mathrm{g}$ ' and ' $\mathrm{d}$ ' in the cells are used to identify whether the $P$-string will be growing or disappearing. Relevant cases are in parentheses.

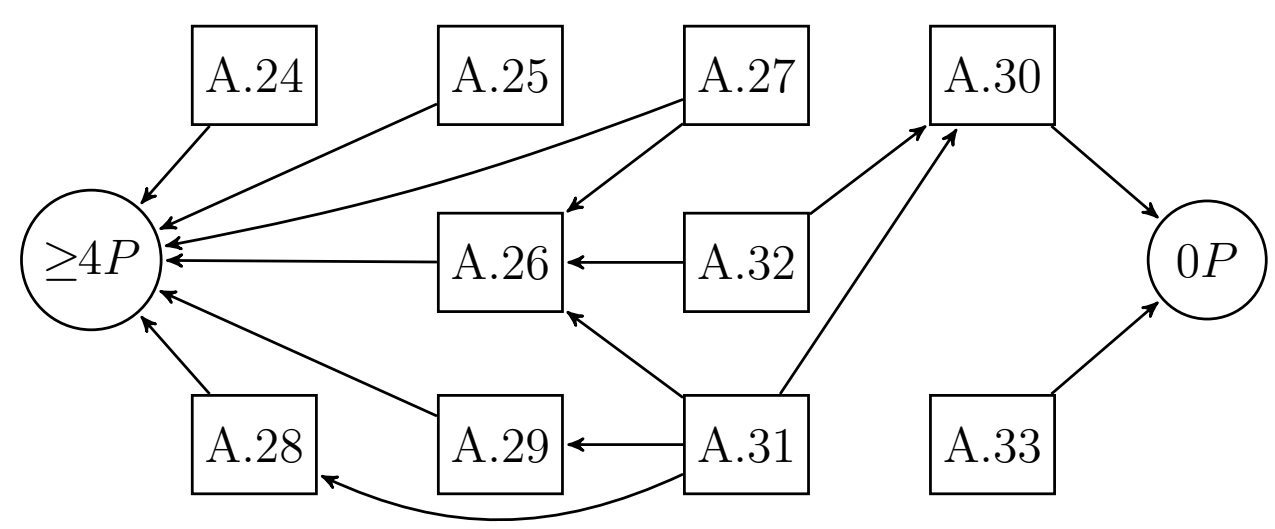

Figure 10: Transforming $3 P$-strings based on the cases specified in the squares results in the $P$-strings in the circles.

\section{A.5 $\geq 4 P$-strings}

Lemma 9. Let $4 \leq k<n$ and let $[a, a+k-1]$ be a $k P$-string of the state $x \in X$. Then one of the following three cases occurs:

(i) there is $y \in F(x) \cup F^{2}(x) \cup F^{3}(x)$ such that $p(y) \leq p(x)$ and $y$ contains a $\geq(k+1) P$-string;

(ii) $k$ is even and there is $y \in F^{k / 2}(x) \cup F^{k / 2+1}(x) \cup F^{k / 2+2}(x)$ such that $p(y) \leq p(x)-1$;

(iii) $k$ is odd and there is $y \in F^{(k+1) / 2}(x) \cup F^{(k+3) / 2}(x)$ such that $p(y) \leq p(x)-1$.

Proof. An agent who is surrounded by agents using the same strategy as him will not change his strategy in one iteration. Therefore, an agent who is not at the very edge of a string 
cannot change his strategy within one iteration. That is why looking only at the ends of a $\geq 4 P$-string in a particular state provides enough information about the string's development in the next iteration. Cases A.34-A.36 below look at the ends of a $\geq 4 P$-string by considering the outermost two agents in the $P$-string who implement the $P$-strategy and their closest neighbors who do not implement it. These three cases along with the auxiliary Cases A.37A.38 constitute the preliminary cases that are needed to construct and describe all possible $\geq 4 P$-strings. This is done in Cases A.39-A.44. Cases A.34 and A.35 will be used in the construction of $\geq 4 P$-strings which grow, while Case A.36 and Cases A.37-A.38 will be used for the construction of $\geq 4 P$-strings which shrink.

Case A.34: $\left(x_{1}, x_{2}, x_{3}\right)=(P, P, A)$

Let $d$ be such that $\left(d_{1}, d_{2}\right)=(R, L)$ and $y=f(d)$. Then $\pi_{1} \geq 1-c, \pi_{2}=2-c$ and $\pi_{3} \leq 1-2 c$.

(a) $d_{4} \in\{A, R, L, E\}$ and $\pi_{4} \leq 2-c$ : then $\left(y_{1}, y_{2}, y_{3}\right)=(P, P, P)$.

(b) $d_{4}=E$ and $\pi_{4}=2$ : then $\left(y_{1}, y_{2}, y_{3}, y_{4}\right)=(P, P, E, E)$.

Case A.35: $\left(x_{1}, x_{2}, x_{3}\right)=(P, P, E)$

Let $d$ be such that $\left(d_{1}, d_{2}\right)=(R, L)$ and $y=f(d)$. Then $\pi_{1} \in\{1-c ; 2-c\}$ and $\pi_{2}=1-c$.

(a) $d_{4} \in\{R, E\}$ : then $\pi_{3}=0$ and $\pi_{4} \leq 1$, hence $\left(y_{1}, y_{2}, y_{3}\right)=(P, P, P)$.

(b) $d_{4} \in\{A, L\}$ and $\pi_{1}=2-c$ : then $\pi_{3}=1$ and $\pi_{4} \leq 1-c$, hence $\left(y_{1}, y_{2}, y_{3}\right)=(P, P, E)$.

(c) $d_{4} \in\{A, L\}$ and $\pi_{1}=1-c$ : then $\pi_{3}=1$ and $\pi_{4} \leq 1-c$, hence $\left(y_{1}, y_{2}, y_{3}\right)=(P, E, E)$.

Case A.36: $\left(x_{1}, x_{2}, x_{3}\right)=(P, P, E)$

Let $d$ be such that $\left(d_{1}, d_{2}\right)=(L, R)$ and $y=f(d)$. Then $\pi_{1} \leq 1-c, \pi_{2}=-c$.

(a) $d_{4} \in\{A, L\}$ : then $\pi_{3}=2$ and $\pi_{4} \leq 1-c$, hence $\left(y_{1}, y_{2}, y_{3}, y_{4}\right)=(P, E, E, E)$.

(b) $d_{4} \in\{E, R\}$ : then $\pi_{3}=1$ and $\pi_{4} \geq 0$, hence $\left(y_{1}, y_{2}, y_{3}\right)=(P, E, E)$.

(c) $\left(d_{4}, d_{5}\right)=(E, E)$ : then $\pi_{3}=1$ and $\pi_{4}=0$, hence $\left(y_{1}, y_{2}, y_{3}, y_{4}\right)=(P, E, E, E)$.

Case A.37: $\left(x_{1}, x_{2}, x_{3}, x_{4}, x_{5}, x_{6}\right)=(E, E, P, P, E, E)$

Let $d$ be such that $\left(d_{3}, d_{4}\right)=(L, R)$ and $y=f(d)$. Then $\pi_{1} \leq 1, \pi_{2}=1, \pi_{3}=-c, \pi_{4}=-c$, $\pi_{5}=1$ and $\pi_{6} \leq 1$, hence $\left(y_{2}, y_{3}, y_{4}, y_{5}\right)=(E, E, E, E)$.

Case A.38: $\left(x_{1}, x_{2}, x_{3}, x_{4}, x_{5}, x_{6}, x_{7}, x_{8}\right)=(E, E, P, P, P, E, E, E)$

Let $d$ be such that $\left(d_{3}, d_{4}, d_{5}\right)=(L, R, R)$ and $y=f(d)$. Then $\pi_{1} \geq 0, \pi_{2}=1, \pi_{3}=-c, \pi_{4}=$ $-c, \pi_{5}=1-c, \pi_{6}=1, \pi_{7}=0$ and $\pi_{8} \geq 0$, hence $\left(y_{2}, y_{3}, y_{4}, y_{5}, y_{6}, y_{7}\right)=(E, E, P, E, P, E)$. Now Case A.10 applies and there is $z \in F(y)$ such that $\left(z_{3}, z_{4}, z_{5}, z_{6}, z_{7}\right)=(E, E, E, E, E)$ 
and $p(z) \leq p(y)-2$.

This concludes the preliminary cases which are needed to handle the ones outlined below. Cases A.39-A.44 that follow explicitly describe the development of every $\geq 4 P$-string that can occur in the current framework using the preliminary cases presented above. Table 8 summarizes the outcomes. It is important to note that in some instances considering the closest one neighboring agent to a $\geq 4 P$-string is sufficient, while in other cases the closest two neighbors are needed. This is specified in every separate case.

Case A.39: $\left(x_{1}, x_{2}, \ldots, x_{k+1}, x_{k+2}\right)=(A, P, \ldots, P, A)$

Take $d_{2}=R, d_{3}=L, d_{k}=R, d_{k+1}=L$, for $i \neq 2,3, k, k+1, d_{i}=d_{i}^{*}$, and $y=$ $f(d)$. If Case A.34(a) applies on at least one of the sides, then the $k P$-string $[2, k+1]$ grows and Lemma 3 implies $p(y) \leq p(x)$. Otherwise, Case A.34(b) applies for both sides, $\left(y_{0}, y_{1}, y_{2}, \ldots, y_{k}, y_{k+1}, y_{k+2}\right)=(E, E, P, \ldots, P, E, E)$, and Lemma 3 implies $p(y) \leq p(x)$. Take $d_{2}^{\prime}=R, d_{3}^{\prime}=L, d_{k}^{\prime}=R, d_{k+1}^{\prime}=L$, for $i \neq 2,3, k, k+1, d_{i}^{\prime}=d_{i}^{*}$, and $z=f\left(d^{\prime}\right)$. According to Case A.35(a), $\left(z_{1}, \ldots, z_{k+1}\right)=(P, \ldots, P)$, the $k P$-string $[2, k+1]$ grows, and Lemma 3 implies $p(z) \leq p(y)$.

Case A.40: $\left(x_{1}, x_{2}, \ldots, x_{k+1}, x_{k+2}\right)=(A, P, \ldots, P, E)$ and $d_{k+3}^{*} \in\{E, R\}$

Take $d_{2}=R, d_{3}=L, d_{k}=R, d_{k+1}=L$, for $i \neq 2,3, k, k+1, d_{i}=d_{i}^{*}$, and $y=f(d)$. By Case A.34 and Case A.35(a), the $k P$-string [2, $k+1]$ grows and Lemma 3 implies $p(y) \leq p(x)$.

Case A.41: $d_{0}^{*} \in\{E, L\},\left(x_{1}, x_{2}, \ldots, x_{k+1}, x_{k+2}\right)=(E, P, \ldots, P, E)$ and $d_{k+3}^{*} \in\{E, R\}$

Take $d_{2}=R, d_{3}=L, d_{k}=R, d_{k+1}=L$, for $i \neq 2,3, k, k+1, d_{i}=d_{i}^{*}$, and $y=f(d)$. According to Case A.35(a), the $k P$-string $[2, k+1]$ grows and Lemma 3 implies $p(y) \leq p(x)$.

Case A.42: $d_{0}^{*} \in\{E, L\},\left(x_{1}, x_{2}, \ldots, x_{k+1}, x_{k+2}\right)=(E, P, \ldots, P, E)$ and $d_{k+3}^{*} \in\{A, L\}$

Define $x^{0}=x$. Take $d_{2}^{0}=L, d_{3}^{0}=R, d_{k}^{0}=L, d_{k+1}^{0}=R$, for $i \neq 2,3, k, k+1, d_{i}^{0}=d_{i}^{*}$, and $x^{1}=f\left(d^{0}\right)$. According to Case A.36(a) and (b), the $k P$-string shrinks from both sides. It holds that $\left(x_{1}^{1}, x_{2}^{1}, x_{3}^{1}, \ldots, x_{k}^{1}, x_{k+1}^{1}, x_{k+2}^{1}, x_{k+3}^{1}\right)=(E, E, P, \ldots, P, E, E, E)$. Lemma 3 implies $p\left(x^{1}\right) \leq p\left(x^{0}\right)$. For $m=1, \ldots,\lfloor(k-4) / 2\rfloor$, define $d_{2+m}^{m}=L, d_{3+m}^{m}=R, d_{k-m}^{m}=L, d_{k-m+1}^{m}=$ $R$, for $i \neq 2+m, 3+m, k-m, k-m+1, d_{i}^{m}=d_{i}^{*}$, and $x^{m+1}=f\left(d^{m}\right)$. According to Case A.36(c), it holds that $x^{m+1}$ has a $(k-2 m-2) P$-string $[3+m, k-m]$ and $x_{1+m}^{m+1}=x_{2+m}^{m+1}=$ $x_{k-m+1}^{m+1}=x_{k-m+2}^{m+1}=x_{k-m+3}^{m+1}=E$. Lemma 3 implies that $p\left(x^{m+1}\right) \leq p\left(x^{m}\right)$. If $k$ is even, then take $d_{k / 2+1}^{k / 2-1}=L, d_{k / 2+1}^{k / 2-1}=R$, for $i \neq k / 2+1, k / 2+2, d_{i}^{k / 2-1}=d_{i}^{*}$, and $x^{k / 2}=f\left(d^{k / 2}-1\right)$. According to Case A.37, it holds that the $2 P$-string $[k / 2+1, k / 2+2]$ disappears and Lemma 3 implies that $p\left(x^{k / 2}\right) \leq p\left(x^{k / 2-1}\right)-1$. If $k$ is odd, then take $d_{(k+1) / 2}^{(k-3) / 2}=L, d_{(k+3) / 2}^{(k-3) / 2}=R$, $d_{(k+5) / 2}^{(k-3) / 2}=R$, for $i \neq(k+1) / 2,(k+3) / 2,(k+5) / 2, d_{i}^{(k-3) / 2}=d_{i}^{*}$, and $x^{(k-1) / 2}=f\left(d^{(k-3) / 2}\right)$. According to Case A.38, it holds that $x^{(k-1) / 2}$ has two $1 P$-strings $\{(k+1) / 2\}$ and $\{(k+5) / 2\}$. Lemma 3 implies that $p\left(x^{(k-1) / 2}\right) \leq p\left(x^{(k-3) / 2}\right)+1$. Moreover, according to Case A.38, there is $x^{(k+1) / 2} \in F\left(x^{(k-1) / 2}\right)$ such that $p\left(x^{(k+1) / 2}\right) \leq p\left(x^{(k-1) / 2}\right)-2$ and the $1 P$-strings $\{(k+1) / 2\}$ 
and $\{(k+5) / 2\}$ disappear.

Case A.43: $d_{0}^{*} \in\{A, R\},\left(x_{1}, x_{2}, \ldots, x_{k+1}, x_{k+2}\right)=(E, P, \ldots, P, E)$ and $d_{k+3}^{*} \in\{A, L\}$ Take $d_{2}=L, d_{3}=R, d_{k}=L, d_{k+1}=R$, for $i \neq 2,3, k, k+1, d_{i}=d_{i}^{*}$, and $y=f(d)$. According to Case A.36(a) the $k P$-string shrinks from both sides. It holds that $\left(y_{1}, y_{2}, y_{3}, \ldots\right.$, $\left.y_{k}, y_{k+1}, y_{k+2}, y_{k+3}\right)=(E, E, P, \ldots, P, E, E, E)$. Lemma 3 implies $p(y) \leq p(x)$. From here, the argument is identical to the one in Case A.42.

Case A.44: $\left(x_{1}, x_{2}, \ldots, x_{k+1}, x_{k+2}\right)=(A, P, \ldots, P, E)$ and $d_{k+3}^{*} \in\{A, L\}$

On the left-hand side either Case A.34(a) or Case A.34(b) applies and on the right-hand side either Case A.35(b) or Case A.35(c) applies. This give rise to the following four possibilities:

(i) Case A.34(a) and Case A.35(b). Take $d_{2}=R, d_{3}=L, d_{k}=R, d_{k+1}=L$, for $i \neq$ $2,3, k, k+1, d_{i}=d_{i}^{*}$, and $y=f(d)$. The $k P$-string $[2, k+1]$ grows and Lemma 3 implies $p(y) \leq p(x)$.

(ii) Case A.34(b) and Case A.35(b). Take $d_{2}=R, d_{3}=L, d_{k}=R, d_{k+1}=L$, for $i \neq 2,3, k, k+1, d_{i}=d_{i}^{*}$, and $y=f(d)$. It holds that $\left(y_{0}, y_{1}, y_{2}, \ldots, y_{k+1}, y_{k+2}\right)=$ $(E, E, P, \ldots, P, E)$ and Lemma 3 implies $p(y) \leq p(x)$. Either Case A.41 or Case A.42 applies. In the former case there is $z \in F(y)$ such that the $k P$-string $[2, k+1]$ grows and Lemma 3 implies $p(z) \leq p(y)$. In the latter case, if $k$ is even there is $x^{k / 2} \in F^{k / 2}(y)$ such that $p\left(x^{k / 2}\right) \leq p(y)-1$ and the $k P$-string $[2, k+1]$ shrinks until it disappears, and if $k$ is odd there is $x^{(k+1) / 2} \in F^{(k+1) / 2}(y)$ such that $p\left(x^{(k+1) / 2}\right) \leq p(y)-1$ and the $k P$-string $[2, k+1]$ shrinks until it disappears.

(iii) Case A.34(a) and Case A.35(c). Take $d_{2}=R, d_{3}=L, d_{k}=R, d_{k+1}=L$, for $i \neq 2,3, k, k+1, d_{i}=d_{i}^{*}$, and $y=f(d)$. It holds that $\left(y_{1}, \ldots, y_{k}, y_{k+1}, y_{k+2}\right)=$ $(P, \ldots, P, E, E)$ and Lemma 3 implies $p(y) \leq p(x)$. If $y_{0}=P$ then $y$ contains a $\geq(k+1) P$ string and we are done, so consider the case where $y_{0} \neq P$. Either Case A.40, Case A.41 or Case A.42 applies. If Case A.40 applies there is $z \in F(y)$ such that the $k P$-string $[1, k]$ grows and Lemma 3 implies $p(z) \leq p(y)$. For the other two cases, the argument is identical to the one in (ii).

(iv) Case A.34(b) and Case A.35(c). Take $d_{2}=R, d_{3}=L, d_{k}=R, d_{k+1}=L$, for $i \neq 2,3, k, k+1, d_{i}=d_{i}^{*}$, and $y=f(d)$. It holds that $\left(y_{0}, y_{1}, y_{2}, \ldots, y_{k}, y_{k+1}, y_{k+2}\right)=$ $(E, E, P, \ldots, P, E, E)$ and Lemma 3 implies $p(y) \leq p(x)$.

We first consider the case $k \geq 5$. Take $d_{2}^{\prime}=R, d_{3}^{\prime}=L, d_{k-1}^{\prime}=R, d_{k}^{\prime}=L$, for $i \neq 2,3, k-1, k, d_{i}^{\prime}=d_{i}^{*}$, and $z=f\left(d^{\prime}\right)$. It holds by Case A.35(a) that $\left(z_{1}, \ldots, z_{k+1}\right)=$ $(P, \ldots, P)$, so the $k P$-string $[2, \ldots, k+1]$ grows and Lemma 3 implies $p(z) \leq p(y)$.

Now consider the case $k=4$. Case A.34(b) implies $\pi_{0}=2$, so $d_{-1} \in\{A, R\}$ and $\pi_{-1} \leq 1-c$, and hence $y_{-1}=E$. Take $d_{2}^{\prime}=R, d_{3}^{\prime}=R, d_{4}^{\prime}=L$, for $i \neq 2,3,4, d_{i}^{\prime}=d_{i}^{*}$, 
and $z=f\left(d^{\prime}\right)$. It holds that $\left(z_{0}, z_{1}, z_{2}, z_{3}, z_{4}, z_{5}\right)=(E, E, P, P, P, P)$ and Lemma 3 implies $p(z) \leq p(y)$. The argument now continues as in (iii).

\begin{tabular}{|c|c|c|c|c|}
\hline \multirow{2}{*}{\multicolumn{2}{|c|}{$\begin{array}{l}\left(d_{3}, \ldots, d_{k+2}\right)= \\
(P, \ldots, P)\end{array}$}} & \multicolumn{3}{|c|}{$\left(d_{k+3}^{*}, d_{k+4}^{*}\right)=$} \\
\hline & & $(A, A / R / L / E)$ & $(E, E / R)$ & $(E, A / L)$ \\
\hline$\overparen{\pi}$ & $(A / R / L / E, A)$ & (A.39) & $(\mathrm{A} .40)$ & $\overline{(\mathrm{A} .44)}$ \\
\hline 8 & $(E / L, E)$ & & (A.41) & $(\mathrm{A} .42)$ \\
\hline 2 & $(A / R, E)$ & & & (A.43) \\
\hline
\end{tabular}

Table 8: Resulting structures for $\geq 4 P$-strings surrounded from the left by the decisions in the columns and from the right by the decisions in the rows. Abbreviations ' $\mathrm{g}$ ' and ' $\mathrm{d}$ ' in the cells are used to identify whether the $P$-string will be growing or disappearing. Relevant cases are in parentheses. 


\section{References}

Ballester, C. and M. Vorsatz (2014). Random walk-based segregation measures. Review of Economics and Statistics 96(3), 383-401.

Bergstrom, T. C. (2002). Evolution of social behavior: Individual and group selection. The Journal of Economic Perspectives 16(2), 67-88.

Bergstrom, T. C. and O. Stark (1993). How altruism can prevail in an evolutionary environment. The American Economic Review 83(2), 149-155.

Ellison, G. (1993). Learning, local interaction, and coordination. Econometrica 61(5), 10471071.

Eshel, I., D. K. Herreiner, L. Samuelson, E. Sansone, and A. Shaked (2000). Cooperation, mimesis, and local interaction. Sociological Methods \& Research 28(3), 341-364.

Eshel, I., L. Samuelson, and A. Shaked (1998). Altruists, egoists, and hooligans in a local interaction model. The American Economic Review 88(1), 157-179.

Eshel, I., E. Sansone, and A. Shaked (1999). The emergence of kinship behavior in structured populations of unrelated individuals. International Journal of Game Theory 28(4), 447463.

Fosco, C. and F. Mengel (2011). Cooperation through imitation and exclusion in networks. Journal of Economic Dynamics and Control 35(5), 641-658.

García-Martínez, J. A. and F. Vega-Redondo (2015). Social cohesion and the evolution of altruism. Games and Economic Behavior 92, 74-105.

Grant, A. M. (2013). Give and take: A revolutionary approach to success. Penguin.

Hindriks, J. and G. D. Myles (2013). Intermediate Public Economics (2nd ed. ed.). Cambridge, MA: MIT Press.

Levine, D. K. and W. Pesendorfer (2007). The evolution of cooperation through imitation. Games and Economic Behavior 58(2), 293-315.

Mäthner, E. and R. Lanwehr (2017). Givers, takers and matchers reciprocity styles and their contribution to organizational behaviour. Gruppe. Interaktion. Organisation. Zeitschrift fr Angewandte Organisationspsychologie (GIO) 48(1), 5-13.

Matros, A. (2012). Altruistic versus egoistic behavior in a public good game. Journal of Economic Dynamics and Control 36(4), 642-656. 
Mengel, F. (2009). Conformism and cooperation in a local interaction model. Journal of Evolutionary Economics 19(3), 397-415.

Nowak, M. A. and R. M. May (1992). Evolutionary games and spatial chaos. Nature 359, 826.

Ross, S. M. (2000). Introduction to probability models (7th ed.). San Diego, CA: HAP, Harcourt/Academic Press.

Young, H. P. (1998). Individual strategy and social structure: an evolutionary theory of institutions. Princeton, NJ: Princeton University Press. 